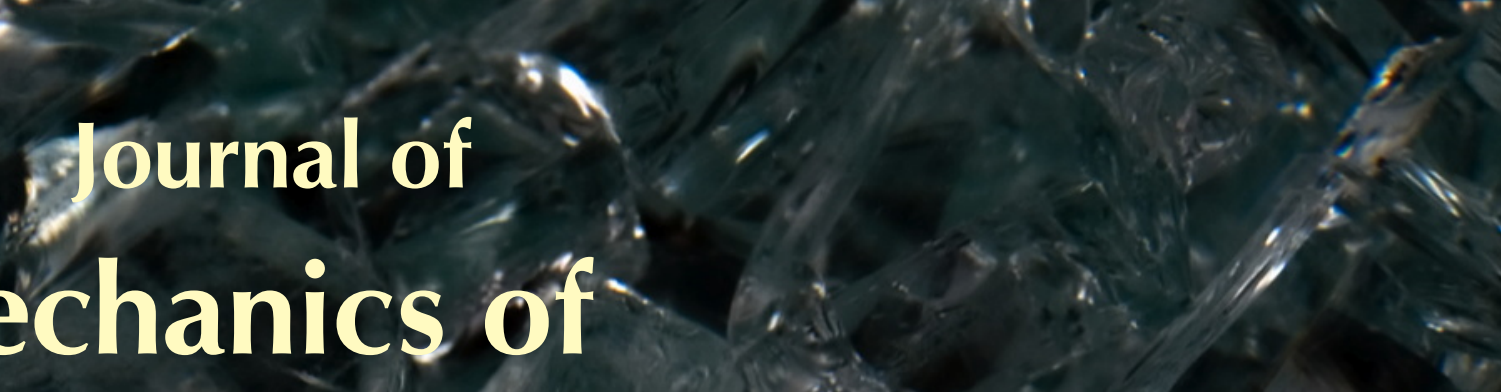

Materials and Structures

Mechanics of

0. 


\title{
NUMERICAL LINEAR STABILITY ANALYSIS OF A THERMOCAPILLARY-DRIVEN LIQUID BRIDGE WITH MAGNETIC STABILIZATION
}

\author{
Yue Huang And BRent C. Houchens
}

\begin{abstract}
A full-zone model of a thermocapillary-driven liquid bridge exposed to a steady, axial magnetic field is investigated using a global spectral collocation method for low-Prandtl number (Pr) fluids. Flow instabilities are identified using normal-mode linear stability analyses. This work presents several numerical issues that commonly arise when using spectral collocation methods and linear stability analyses in the solution of a wide range of partial differential equations. In particular, effects such as discontinuous boundary condition regularization, identification of spurious eigenvalues, and the use of pseudospectra to investigate the robustness of the stability analysis are addressed. Physically, this work provides simulations in the practical range of experimentally utilized magnetic field stabilization in optically heated float-zone crystal growth. A second-order vorticity transport formulation enables modeling of the liquid bridge up to these intermediate magnetic field strength ranges, measured by the Hartmann number (Ha). The thermocapillary driving and magnetic stabilization effects are observed up to $\mathrm{Ha}=500$ for $\mathrm{Pr}=0.001$ and up to $\mathrm{Ha}=300$ for $\mathrm{Pr}=0.02$. Prandtl number effects on temperature and flow fields are investigated within $\operatorname{Pr} \in\left(10^{-12}, 0.0667\right)$ and indicate that $\operatorname{Pr}=0.001$ is a good representation of the base state in the $\mathrm{Pr} \rightarrow 0$ limit, at least up to $\mathrm{Ha}=300$.
\end{abstract}

\section{Introduction}

Float-zone growth processes are methods to grow crystals with the highest purity. A cross-sectional region of a polycrystalline ingot is melted by lateral heating, for example, in an optical heating furnace [Eyer et al. 1979]. This molten region holds itself from spilling by surface tension, forming a liquid bridge between the feed rod and the grown crystal. As the liquid bridge moves through the furnace, the melt resolidifies as a single crystal if properly controlled. Throughout the process the melt never contacts a crucible, and therefore the grown crystal has very low oxygen contamination. Sufficient heat input is required to avoid the onset of morphological instability at the solidification front [Davis 1993]. The thermocapillary effect at the free surface drives a flow within the liquid bridge. This flow is susceptible to instabilities, which result in structural imperfections in grown crystals and uneven dopant distribution for doped crystals [Eyer et al. 1985; Cröll et al. 1994]. Therefore stabilization techniques are typically used in crystal growth practice. For example, two silicon crystal rods were grown in a double ellipsoidal mirror furnace with rod rotation in Spacelab-1 [Martinez and Eyer 1986]. A review of liquid bridge stabilization strategies can be found in [Lappa 2005b]. One strategy for molten semiconductors, which have properties similar to liquid metals, is to apply external magnetic fields to control flow motion.

This work was supported by the U.S. Air Force Office of Scientific Research.

Keywords: magnetic stabilization, thermocapillary, liquid bridge, linear stability, regularization, pseudospectra. 
The optically heated liquid bridge has been studied by the crystal growth community using simpler models such as the half-zone model, resembling one half of a liquid bridge, and the full-zone model. The liquid bridge has a barrel shape in microgravity and a sagged shape under terrestrial conditions. These free-surface shapes have been simulated in both half-zone [Morthland and Walker 1996] and full-zone [Lappa 2004] models, respectively. In [Nakamura et al. 1998], $m=1$ and 2 oscillating instability modes were observed in molten silicon in an optically heated half-zone configuration on the TR-IA rocket. The hydrodynamic and hydrothermal instability mechanisms of low and high-Pr liquid bridges, respectively, have been confirmed in [Chen et al. 1997; Lappa 2005a; Bouizi et al. 2007] and elsewhere. Lan and Yeh [2004; 2005] performed quite complete full-zone modeling involving three-dimensional radiation, a deformable free surface and melting interfaces, dopant distribution, and axial and transverse magnetic damping. Prange et al. [1999] studied the half-zone instability with axial magnetic field stabilization up to $\mathrm{Ha}=25$.

This work presents a full-zone liquid bridge model with magnetic stabilization, with a focus on the numerical methods and analyses utilized. The goals of this paper are twofold: first, to provide insight into magnetohydrodynamic control in the liquid bridge problem which will aid in the design of floatzone crystal growth experiments and, second, to demonstrate through example the treatment of several common numerical issues, such as regularization, identification of spurious eigenvalues, and sensitivity of linear stability analyses as quantified by pseudospectral analysis, techniques relevant to a wide array of numerical analysis studies.

\section{Problem description}

2A. Full-zone model of a liquid bridge. A liquid bridge of a molten semiconductor is bounded by top and bottom solid boundaries (Figure 1). Both boundaries are assumed flat, electrically insulating, and at the melting temperature $T_{0}^{*}$ of the semiconductor. The lateral cylindrical free surface is assumed nondeformable due to high surface tension and the microgravity environment. The diameter and height

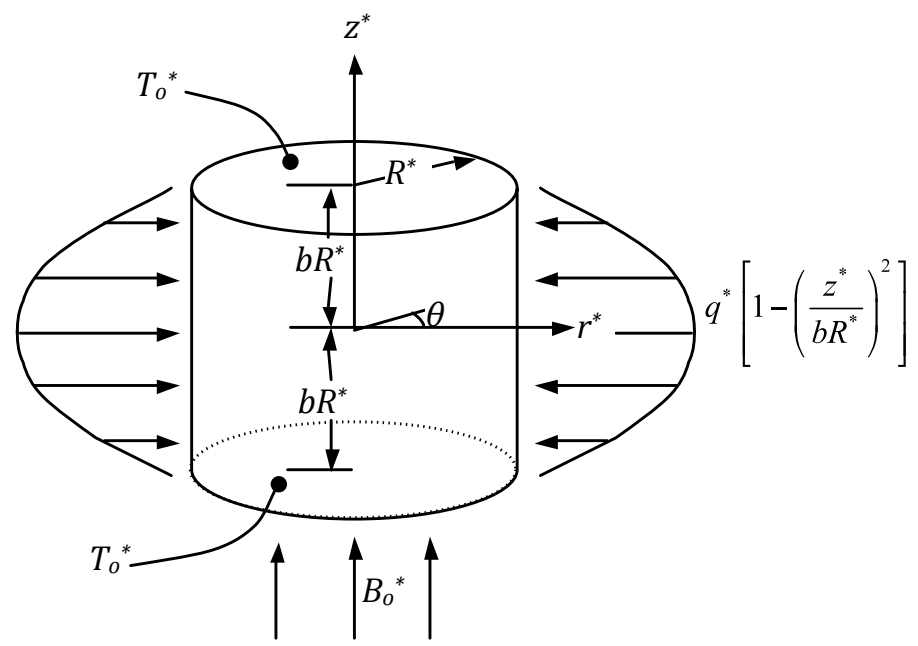

Figure 1. Full-zone model of a liquid bridge with a parabolic heat flux at the free surface and an axially applied magnetic field. 
of the liquid bridge are $2 R^{*}$ and $2 b R^{*}$, respectively. The aspect ratio $b$ is held at 1 in this paper. An axisymmetric heat flux is applied at the lateral free surface. This heat flux is approximated with a maximum intensity $q^{*}$ at the equatorial plane and parabolic reduction to zero at the top and bottom boundaries. The thermal conductivity of the melt is $k^{*}$. A constant, uniform external magnetic field is applied in the axial direction with a magnetic flux density of $\boldsymbol{B}^{*}=B_{o}^{*} \hat{\boldsymbol{e}}_{z}$. Variables with an asterisk superscript are dimensional quantities.

The characteristic length, temperature, and magnetic flux density are $R^{*}, \Delta T^{*}=q^{*} R^{*} / k^{*}$, and $B_{o}^{*}$, respectively. Temperature is scaled as $T=\left(T^{*}-T_{0}^{*}\right) / \Delta T^{*}$. The nondimensional governing equations are incompressible continuity, the Navier-Stokes equations including the electromagnetic body force, the energy equation neglecting viscous dissipation, conservation of charge, and Ohm's law:

$$
\begin{gathered}
\nabla \cdot \boldsymbol{v}=0 \\
\frac{\partial \boldsymbol{v}}{\partial t}+(\boldsymbol{v} \cdot \nabla) \boldsymbol{v}=-\nabla P+\nabla^{2} \boldsymbol{v}+\mathrm{Ha}^{2}\left(\boldsymbol{j} \times \hat{\boldsymbol{e}}_{z}\right), \\
\operatorname{Pr}\left[\frac{\partial T}{\partial t}+(\boldsymbol{v} \cdot \nabla) T\right]=\nabla^{2} T, \\
\nabla \cdot \boldsymbol{j}=0, \\
\boldsymbol{j}=-\nabla \phi+\boldsymbol{v} \times \hat{\boldsymbol{e}}_{z},
\end{gathered}
$$

where

$$
\mathrm{Ha}=\left(\frac{\sigma^{*}}{\mu^{*}}\right)^{\frac{1}{2}} B_{o}^{*} R^{*}, \quad \operatorname{Pr}=\frac{\mu^{*} c_{p}^{*}}{k^{*}} .
$$

The Hartmann number Ha is proportional to the magnetic flux density $B_{o}^{*}$ and measures the ratio of the electromagnetic body forces to the viscous forces. The Prandtl number Pr indicates the relative effectiveness of thermal convection to heat conduction in the melt. Pr is a material property, where $c_{p}^{*}$ is the specific heat of the melt. For fluids with small Prandtl number (for example, $\operatorname{Pr}_{\text {silicon }} \approx 0.02$ ), heat conduction is dominant over convection.

Nondimensional boundary conditions include a nondeformable and electrically insulating free surface ( $v_{r}=0$ and $j_{r}=0$ at $r=1$ ) with flow induced by the thermocapillary boundary conditions, (2-9), and no-slip, no-penetration and electrically insulating top and bottom boundaries $\left(\boldsymbol{v}=0\right.$ and $j_{z}=0$ at $\left.z= \pm b\right)$ which are maintained at the melting temperature $(T=0$ at $z= \pm b)$.

The full-zone is a more realistic liquid bridge model for optically heated float-zone crystal growth as compared to the half-zone, though both capture much of the primary physics of the flow field. In the full-zone model the heat flux is input on the free surface, rather than from a hot bottom wall, as in the half-zone. Also, no constraint is enforced at the midplane in the full-zone. Thus the temperature varies at the midplane (see Figures $4 \mathrm{a}$ and $4 \mathrm{~b}$ ) and flow is allowed to be nonzero and even cross the midplane (see Figure 6). Note that in this work axial symmetry is assumed in the base flow for computational efficiency, but no boundary condition is imposed at the midplane. Therefore the full-zone character is maintained.

In contrast, the half-zone intends to model one half of the liquid bridge. A no-slip, no-penetration solid boundary at fixed temperature is enforced in the half-zone, at the location of the midplane. A thermally insulating free surface is commonly assumed. The half-zone is driven by the temperature difference 
between two bounding disks. Despite these simplifications, the half-zone is an effective liquid bridge model for both experimental and computational studies. However, the onset of flow instabilities tend to be delayed in the half-zone by the presence of the no-slip boundary that replaces the midplane and removes momentum from the flow through viscous effects. An extensive comparison of the half-zone and full-zone models can be found in [Houchens and Walker 2005].

2B. Thermocapillary-driven flow. The temperature gradient at the free surface produces surface tension differences, called the thermocapillary or Marangoni effect. The surface tension $\gamma^{*}$ drives a flow within the liquid bridge, and is approximated as a linearly decreasing function of temperature:

$$
\gamma^{*}\left(T^{*}\right)=\gamma_{0}^{*}+\frac{d \gamma^{*}}{d T^{*}}\left(T^{*}-T_{0}^{*}\right),
$$

where $d \gamma^{*} / d T^{*}$ is a negative quantity. With the Newtonian constitutive relations

$$
\tau_{r z}^{*}=\mu^{*}\left(\frac{\partial v_{z}^{*}}{\partial r^{*}}+\frac{\partial v_{r}^{*}}{\partial z^{*}}\right), \quad \tau_{r \theta}^{*}=\mu^{*}\left[r^{*} \frac{\partial}{\partial r^{*}}\left(\frac{v_{\theta}^{*}}{r^{*}}\right)+\frac{1}{r^{*}} \frac{\partial v_{r}^{*}}{\partial \theta}\right],
$$

the nondimensional thermocapillary boundary conditions become

$$
\frac{\partial v_{z}}{\partial r}=-\operatorname{Re}_{\mathrm{FZ}} \frac{\partial T}{\partial z} \quad \text { at } \quad r=1, \quad \frac{\partial v_{\theta}}{\partial r}-\frac{v_{\theta}}{r}=-\operatorname{Re}_{\mathrm{FZ}} \frac{1}{r} \frac{\partial T}{\partial \theta} \quad \text { at } \quad r=1,
$$

where

$$
\operatorname{Re}_{\mathrm{FZ}}=\frac{\left|\frac{d \gamma^{*}}{d T^{*}}\right| \Delta T^{*}}{\mu^{*} V_{c}^{*}}=\frac{\rho^{*} R^{*}\left|\frac{d \gamma^{*}}{d T^{*}}\right| \Delta T^{*}}{\mu^{* 2}} .
$$

The thermocapillary Reynolds number $\mathrm{Re}_{\mathrm{FZ}}$ measures the thermocapillary effect. The subscript FZ refers to the full-zone model temperature scaling. The related Marangoni number $\mathrm{Ma}=\operatorname{Re}_{\mathrm{FZ}} \times \operatorname{Pr}$ is also commonly used in thermocapillary flow studies. The viscous Reynolds number is

$$
\operatorname{Re}_{\mathrm{viscous}}=\frac{\rho^{*} v_{\max }^{*} R^{*}}{\mu^{*}}=\frac{\rho^{*} v_{\max } V_{c}^{*} R^{*}}{\mu^{*}}=v_{\max },
$$

where $V_{c}^{*}=\mu^{*} /\left(\rho^{*} R^{*}\right)$ is the characteristic flow velocity. Thus the maximum dimensionless velocity $v_{\max }$ is equivalent to the viscous Reynolds number in this scaling.

Figure 2 shows the thermocapillary-driven flow in $r \in(0,1), z \in(0, b)$ at some $\theta$ plane. From $z=0 \rightarrow 1$ the temperature decreases along the free surface $(r=1)$, and therefore the surface tension increases. The thermocapillary effect pulls fluid from the equatorial plane toward the top boundary along the free surface. This flow then hits the top boundary, turns inwards and circulates back to the equatorial plane in the interior of the liquid bridge.

\section{Steady axisymmetric base flow}

3A. Base flow assumptions. Periodic and/or three-dimensional flow in the liquid bridge results in imperfections in grown crystals such as striations and nonuniform dopant distribution. With sufficient magnetic stabilization, the base flow is steady $(\partial / \partial t=0)$, axisymmetric $(\partial / \partial \theta=0)$ with zero azimuthal velocity 

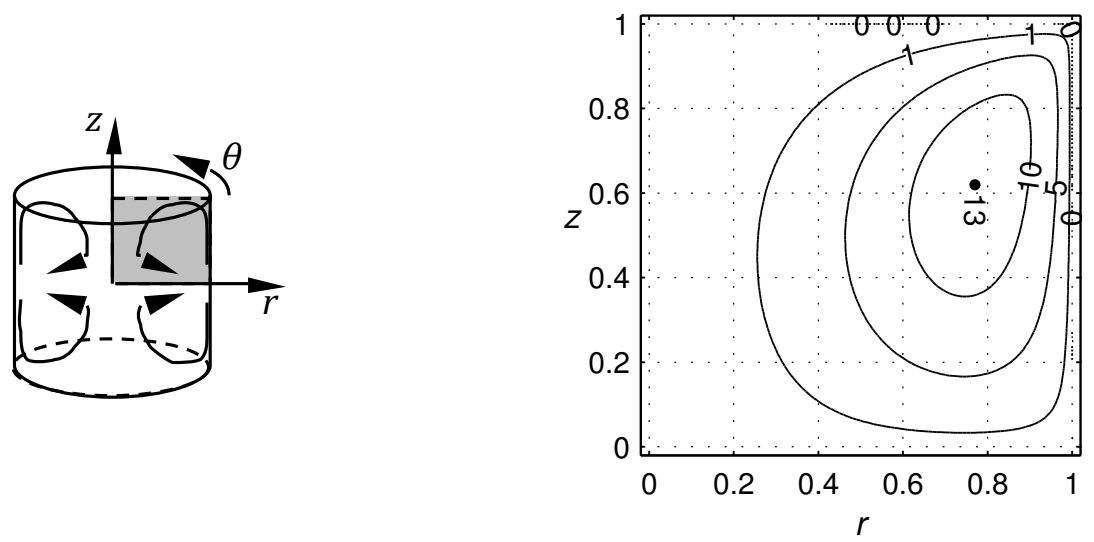

Figure 2. Stream function contours of the thermocapillary-driven flow $(\operatorname{Pr}=0.02$, $\mathrm{Ha}=0$, at the critical $\operatorname{Re}_{\mathrm{FZ}}=1546.58$, flow grid $r \times z=35 \times 45$, temperature grid $r \times z=30 \times 30, \alpha=300$ ).

$\left(v_{\theta 0}=0\right)$, and exhibits axial symmetry about the equatorial plane. For this base flow state the governing equations simplify to

$$
\begin{aligned}
\frac{\partial v_{r 0}}{\partial r}+\frac{v_{r 0}}{r}+\frac{\partial v_{z 0}}{\partial z} & =0, \\
v_{r 0} \frac{\partial v_{r 0}}{\partial r}+v_{z 0} \frac{\partial v_{r 0}}{\partial z} & =-\frac{\partial P_{0}}{\partial r}+\frac{\partial^{2} v_{r 0}}{\partial r^{2}}+\frac{1}{r} \frac{\partial v_{r 0}}{\partial r}-\frac{v_{r 0}}{r^{2}}+\frac{\partial^{2} v_{r 0}}{\partial z^{2}}-\mathrm{Ha}^{2} v_{r 0}, \\
v_{r 0} \frac{\partial v_{z 0}}{\partial r}+v_{z 0} \frac{\partial v_{z 0}}{\partial z} & =-\frac{\partial P_{0}}{\partial z}+\frac{\partial^{2} v_{z 0}}{\partial r^{2}}+\frac{1}{r} \frac{\partial v_{z 0}}{\partial r}+\frac{\partial^{2} v_{z 0}}{\partial z^{2}}, \\
\operatorname{Pr}\left(v_{r 0} \frac{\partial T_{0}}{\partial r}+v_{z 0} \frac{\partial T_{0}}{\partial z}\right) & =\frac{\partial^{2} T_{0}}{\partial r^{2}}+\frac{1}{r} \frac{\partial T_{0}}{\partial r}+\frac{\partial^{2} T_{0}}{\partial z^{2}},
\end{aligned}
$$

with boundary conditions

$$
\begin{aligned}
& v_{r 0}=0, \quad j_{r 0}=0, \quad \frac{\partial v_{z 0}}{\partial r}=-\operatorname{Re}_{\mathrm{FZ}} \frac{\partial T_{0}}{\partial z} F(z), \quad \frac{\partial T_{0}}{\partial r}=1-\left(\frac{z}{b}\right)^{2} \quad \text { at } r=1, \\
& v_{r 0}=0, \quad v_{z 0}=0, \quad T_{0}=0, \quad j_{z 0}=0 \quad \text { at } z= \pm b,
\end{aligned}
$$

where

$$
F(z)=1-\exp \left\{-\alpha\left[1-\left(\frac{z}{b}\right)^{2}\right]^{2}\right\}
$$

is a regularization function to remove the $\partial v_{z 0} / \partial r$ singularity at $(r, z)=(1, \pm b)$ between the thermocapillary driving force on the free surface and the no-penetration conditions at the top and bottom boundaries.

Base flow variables are denoted with subscript 0 to differentiate them from perturbation variables (which have subscript 1).

3B. Second-order vorticity transport formulation. In [Houchens and Walker 2005], a fourth-order stream function formulation was introduced for the base flow problem, with the stream function $\psi$ defined as

$$
v_{r 0}=\frac{1}{r} \frac{\partial \psi}{\partial z}, \quad v_{z 0}=-\frac{1}{r} \frac{\partial \psi}{\partial r} .
$$


The resulting equations were discretized using a global spectral collocation method with Chebyshev basis functions. In this formulation, the momentum equations can be combined into a single fourth-order partial differential equation governing $\psi$. Unfortunately, the coefficients on the derivatives of the Chebyshev functions increase dramatically as the derivative order increases. From a numerical standpoint, the combination of order-one coefficients on the first and second derivatives and huge third and fourth derivatives in one equation causes numerical difficulties. This imbalance in fact imposes a constraint on the maximum viable grid resolution in obtaining converged solutions. This consequently limited the maximum magnetic stabilization intensity (Ha) that could be studied [Houchens and Walker 2001].

Reducing the maximum Chebyshev derivative order improves the numerical performance, at the expense of more dependent variables. In this work, this is achieved by introducing the azimuthal vorticity $\omega_{\theta}$ into the formulation as

$$
\omega_{\theta}=\frac{\partial v_{r 0}}{\partial z}-\frac{\partial v_{z 0}}{\partial r} .
$$

The nondimensional governing equations of the second-order vorticity transport formulation are

$$
\begin{aligned}
\omega_{\theta} & =\frac{1}{r} \frac{\partial^{2} \psi}{\partial r^{2}}-\frac{1}{r^{2}} \frac{\partial \psi}{\partial r}+\frac{1}{r} \frac{\partial^{2} \psi}{\partial z^{2}} \\
\frac{1}{r} \frac{\partial \psi}{\partial z}\left[\frac{\partial \omega_{\theta}}{\partial r}-\frac{\omega_{\theta}}{r}\right]-\frac{1}{r} \frac{\partial \psi}{\partial r}\left[\frac{\partial \omega_{\theta}}{\partial z}\right] & =\frac{\partial^{2} \omega_{\theta}}{\partial r^{2}}+\frac{1}{r} \frac{\partial \omega_{\theta}}{\partial r}+\frac{\partial^{2} \omega_{\theta}}{\partial z^{2}}-\frac{\omega_{\theta}}{r^{2}}-\frac{\mathrm{Ha}^{2}}{r} \frac{\partial^{2} \psi}{\partial z^{2}}, \\
\operatorname{Pr}\left(\frac{1}{r} \frac{\partial \psi}{\partial z} \frac{\partial T_{0}}{\partial r}-\frac{1}{r} \frac{\partial \psi}{\partial r} \frac{\partial T_{0}}{\partial z}\right) & =\frac{\partial^{2} T_{0}}{\partial r^{2}}+\frac{1}{r} \frac{\partial T_{0}}{\partial r}+\frac{\partial^{2} T_{0}}{\partial z^{2}},
\end{aligned}
$$

with boundary conditions

$$
\begin{aligned}
& \psi=0, \quad \frac{\partial^{2} \psi}{\partial r^{2}}-\frac{\partial \psi}{\partial r}=\operatorname{Re}_{\mathrm{FZ}} \frac{\partial T_{0}}{\partial z} F(z), \quad \frac{\partial T_{0}}{\partial r}=1-\left(\frac{z}{b}\right)^{2}, \quad \text { at } \quad r=1, \\
& \frac{\partial \psi}{\partial z}=0, \quad \psi=0, \quad T_{0}=0, \quad \text { at } \quad z=b .
\end{aligned}
$$

The base flow variables are represented with Chebyshev polynomials:

$$
\begin{aligned}
\psi\left(r, \frac{z}{b}\right) & =r^{2} \sum_{L=0}^{N_{R F}+1} \sum_{M=0}^{N_{Z F}+1} A_{L M} T_{2 L}(r) T_{2 M+1}\left(\frac{z}{b}\right), \\
\omega_{\theta}\left(r, \frac{z}{b}\right) & =r \sum_{L=0}^{N_{R F}} \sum_{M=0}^{N_{Z F}} B_{L M} T_{2 L}(r) T_{2 M+1}\left(\frac{z}{b}\right), \\
T_{0}\left(r, \frac{z}{b}\right) & =\sum_{L=0}^{N_{R T}} \sum_{M=0}^{N_{Z T}} C_{L M} T_{2 L}(r) T_{2 M}\left(\frac{z}{b}\right),
\end{aligned}
$$

where $T_{n}(r)=\cos (n \arccos r)$ are the Chebyshev basis functions. As a result of radial symmetry (axisymmetry), only even Chebyshev terms are utilized in $r$, with the overall radial symmetry set by the multiple of $r$ in front of the representation. This corresponds to the behaviors as $r \rightarrow 0$, which were investigated for $\psi, \omega_{\theta}$, and $T$ using the Frobenius method. Moreover, due to the axial symmetry, only even or odd Chebyshev terms are nonzero in $z$. Taking advantage of axisymmetry and the axial symmetry, the unique 
computational domain for the base flow is reduced to $r \in(0,1), z \in(0, b)$ at $\theta=0$ and is discretized using Gauss-Lobatto collocation grids $\left(N_{R F} \times N_{Z F}\right.$ for the flow field and $N_{R T} \times N_{Z T}$ for the temperature field) given, for example, by

$$
\begin{array}{rlrl}
r_{I F} & =\cos \frac{I_{F} \pi}{2 N_{R F}} & \text { for } & I_{F}=0,1,2, \ldots, N_{R F}, \\
z_{K F}=\cos \frac{K_{F} \pi}{2 N_{Z F}} & \text { for } & K_{F}=0,1,2, \ldots, N_{Z F} .
\end{array}
$$

These grids weight the finest resolution toward the free surface and the solid boundary where it is most needed.

At $r=0$ or $z=0$, the governing equations (3-10) and (3-11) and the first two boundary conditions in (3-13) (at $z=0$ ) reduce to $0=0$. To impose constraints at $r=0$ and $z=0$, the first nonzero leading order of these equations are applied. For example, after substituting the representations for $\psi$ and $\omega_{\theta}$ from (3-15) and (3-16), respectively, (3-10) becomes

$$
\sum_{L=0}^{N_{R F}} \sum_{M=0}^{N_{Z F}} B_{L M} r T_{2 L} T_{2 M+1}-\sum_{L=0}^{N_{R F}+1} \sum_{M=0}^{N_{Z F}+1} A_{L M} \frac{r}{b^{2}} T_{2 L} T_{2 M+1}^{\prime \prime}-\sum_{L=0}^{N_{R F}+1} \sum_{M=0}^{N_{Z F}+1} A_{L M}\left(r T_{2 L}^{\prime \prime}+3 T_{2 L}^{\prime}\right) T_{2 M+1}
$$

Equation (3-20) simplifies to $0=0$ at $r=0$. Instead, if the Chebyshev basis functions are expanded in their Taylor series

$$
T_{2 L}(r)=(-1)^{L}\left[1-2 L^{2} r^{2}+\frac{2}{3} L^{2}\left(L^{2}-1\right) r^{4}+O\left(r^{6}\right)\right],
$$

the coefficients of the nonzero leading order $\left(r^{1}\right)$ can be used as a constraint at $r=0$ :

$$
\begin{aligned}
\sum_{L=0}^{N_{R F}} \sum_{M=0}^{N_{Z F}} B_{L M} \not{ }^{\prime}(-1)^{L} T_{2 M+1} & -\sum_{L=0}^{N_{R F}+1} \sum_{M=0}^{N_{Z F}+1} A_{L M} \frac{\not h^{\prime}}{b^{2}}(-1)^{L} T_{2 M+1}^{\prime \prime} \\
& -\sum_{L=0}^{N_{R F}+1} \sum_{M=0}^{N_{Z F+1}} A_{L M}\left[\not \gamma(-1)^{L}\left(-4 L^{2}\right)+3(-1)^{L}\left(-4 L^{2} \not r\right)\right] T_{2 M+1}=0 .
\end{aligned}
$$

It was found that the constraints above at $r=0$ and $z=0$ enhance the accuracy of the solution greatly with minimal additional computational cost. Therefore these constraints were applied in all cases presented here. At the top boundary $z=b$, boundary conditions were applied using orthogonality, hence special treatment was not required at $(r, z)=(0, b)$

The $\operatorname{Re}_{\mathrm{FZ}}$ is ramped up starting from $\operatorname{Re}_{\mathrm{FZ}}<1$, where the flow field is almost stagnant, until the desired value is obtained. Solutions for lower $\mathrm{Re}_{\mathrm{FZ}}$ cases become initial guesses for larger $\operatorname{Re}_{\mathrm{FZ}}$ cases. The governing equations and boundary conditions are solved using the Newton-Raphson iterative method. LU decomposition and back substitution is performed using the DGESV routine [Intel 2008] in LAPACK [Anderson et al. 1999]. The base flow code is written in Fortran 90.

3C. Magnetic damping. When exposed to an axial static magnetic field, radial and azimuthal flow motions are damped by the electromagnetic body force. Figure 3 demonstrates this magnetic damping effect by varying the Hartmann number Ha while keeping the material properties (Pr) and the heat input $\left(\operatorname{Re}_{\mathrm{FZ}}\right)$ fixed. 


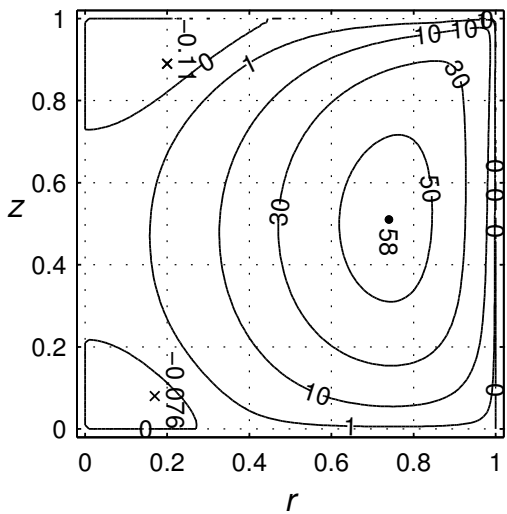

(a) $\mathrm{Ha}=0$

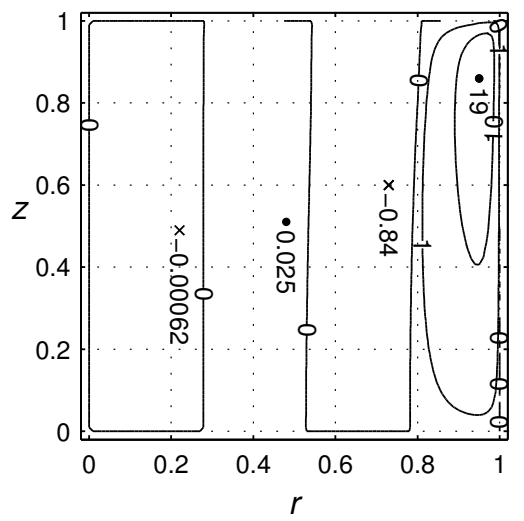

(d) $\mathrm{Ha}=100$

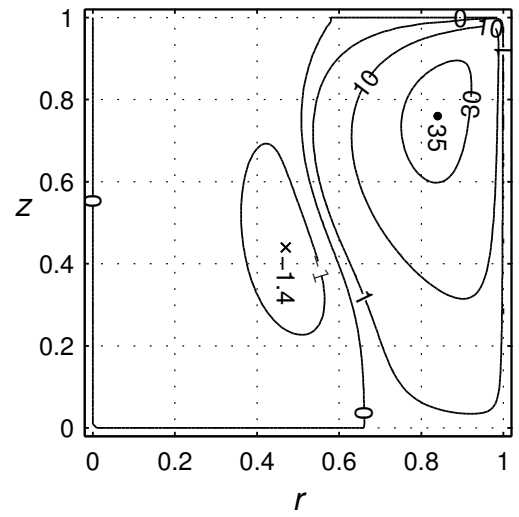

(b) $\mathrm{Ha}=25$

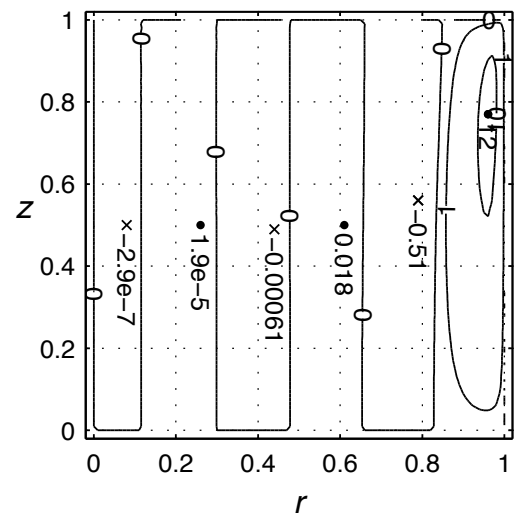

(e) $\mathrm{Ha}=200$

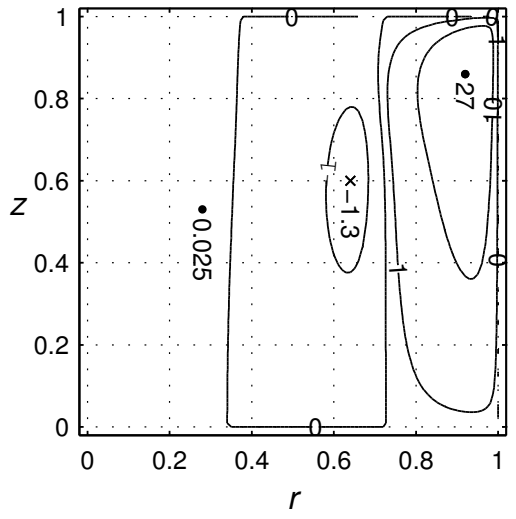

(c) $\mathrm{Ha}=50$

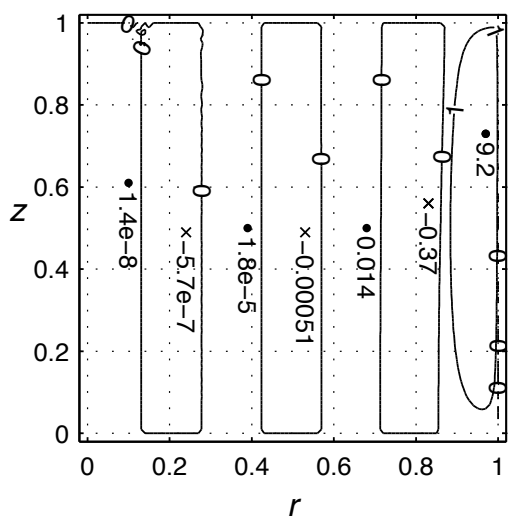

(f) $\mathrm{Ha}=300$

Figure 3. Magnetic damping effect on the stream function contours $\left(\operatorname{Pr}=0.02, \operatorname{Re}_{\mathrm{FZ}}=21,914.8\right)$.

Without magnetic damping $(\mathrm{Ha}=0)$, a primary circulation "cell" forms due to the thermocapillary effect. Flow circulates counterclockwise throughout $r \in(0,1)$ in the upper half of the liquid bridge. Small secondary recirculation cells also emerge in the interior due to strong convection. At $\mathrm{Ha}=25$, the electromagnetic effect weakens the primary circulation cell and confines it within $r \in(0.5,1)$. When exposed to a magnetic field in the $+z$ direction, radial inflow (in the $-r$ direction) near the $(r, z)=(1,1)$ corner induces electric current in the $+\theta$ direction (into the page). This electric current leads to the Lorentz force exerted in the $+r$ direction, which opposes the inflow moving in the $-r$ direction. By continuity, the flow is turned downward and then circulates back as a loop.

As the magnetic field further intensifies, the primary circulation cell is confined more dramatically near the free surface, and more circulation cells develop in the interior. The most significant flow is always within the primary circulation cell. Extremum stream function values within each cell show that the interior of the liquid bridge is effectively stagnant, which is ideal for crystal growth from a melt.

Note that in this example $\operatorname{Re}_{\mathrm{FZ}}$ is chosen at 21,914.8, which is near the critical value for $\operatorname{Pr}=0.02$ at $\mathrm{Ha}=50$. This $\mathrm{Re}_{\mathrm{FZ}}$ value is well above the critical instability values for $\mathrm{Ha}=0$ and $\mathrm{Ha}=25$; therefore the actual flow would be perturbed from the base flow states shown here. 
Isotherms

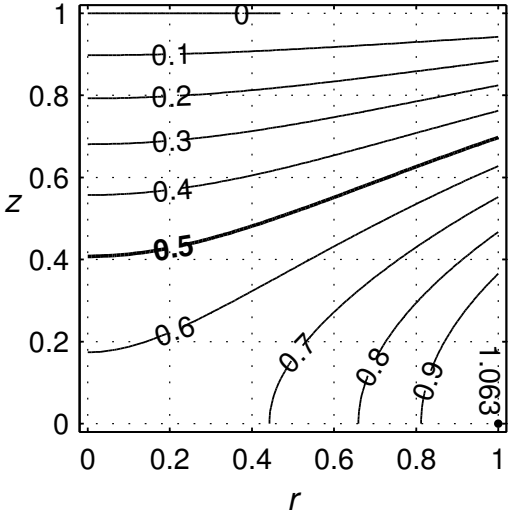

(a) $\operatorname{Pr}=10^{-6}$

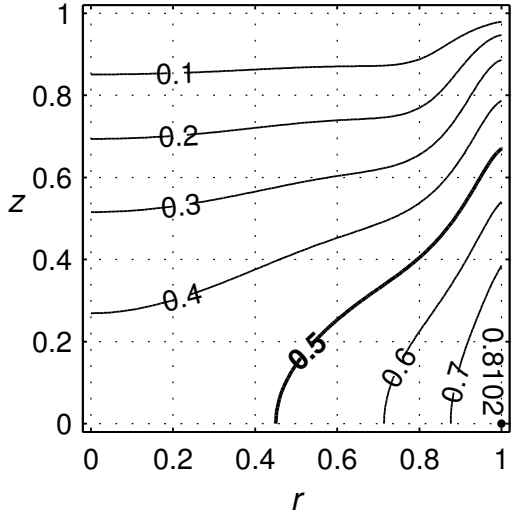

(b) $\operatorname{Pr}=0.0667$

Stream function contours

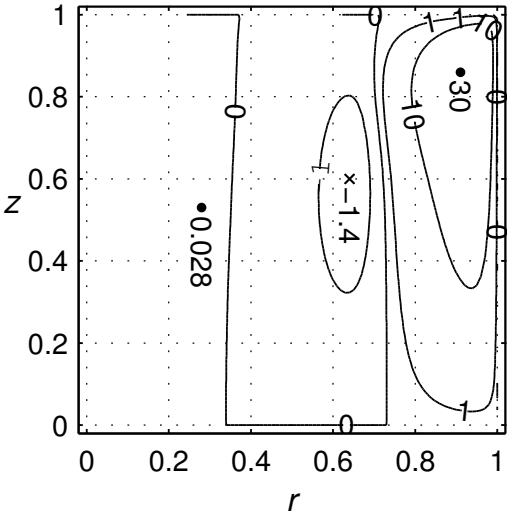

(c) $\operatorname{Pr}=10^{-6}$

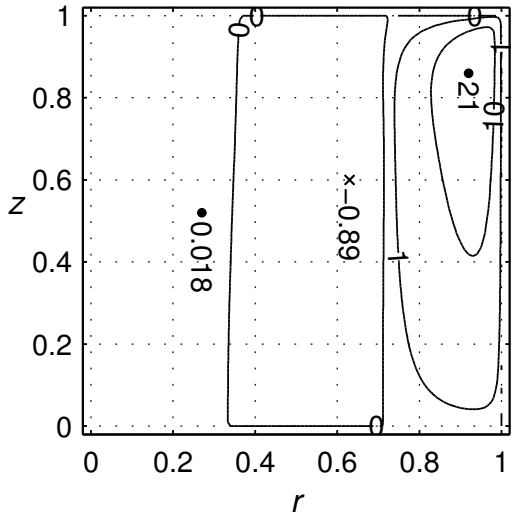

(d) $\operatorname{Pr}=0.0667$

Figure 4. Prandtl number effect on the temperature field and the flow field $\left(\mathrm{Ha}=50, \mathrm{Re}_{\mathrm{FZ}}=21,914.8\right)$.

3D. Prandt number effect. The crystal growth community has frequently studied the effect of Prandtl number Pr, representing different physical materials, in various liquid bridges, typically without magnetic stabilization. For example, Kasperski et al. [2000] first investigated the different characteristics of low and high-Pr full-zone instabilities. Levenstam et al. [2001] performed a fairly continuous Pr study using the half-zone with a focus on the intermediate Pr range which bridges the gap between the low and high-Pr regimes. Bouizi et al. [2007] presented full-zone instabilities over a wide range of $\operatorname{Pr} \in(0.001,100)$ by three-dimensional nonlinear spectral computations.

For a liquid bridge with small Pr, conduction is dominant over convective heat transfer. In the limit of $\operatorname{Pr} \rightarrow 0$, the temperature field is decoupled from the flow field. For example, the isotherms for $\operatorname{Pr}=10^{-6}$ (Figure 4a) indicate pure conduction. In this case $T_{\max }=1.0633$ remains constant as the magnetic field is varied over $\mathrm{Ha} \in(0,300)$ (not shown). $T_{\max }$ is always located at $(r, z)=(1,0)$, the location of maximum heat flux. 
As Pr increases, $T_{\max }$ is reduced due to convective heat transfer (at a fixed $\mathrm{Ha}$ ) assisting conduction in distributing the thermal energy throughout the domain. At $\operatorname{Pr}=0.0667$, isotherms are bent by convection (Figure $4 \mathrm{~b}$ ). Notice that the $T=0.5$ curve shifts by a large amount compared to the case of $\operatorname{Pr}=10^{-6}$.

Compared to the temperature distribution, the Prandtl number has a less significant impact on the flow field with magnetic stabilization. In moving from $\operatorname{Pr}=10^{-6}$ to $\operatorname{Pr}=0.0667$, the flow is weakened (see Figures $4 \mathrm{c}$ and $4 \mathrm{~d}$ ) due to the weaker thermocapillary driving force resulting from the smaller temperature gradient at the free surface. Otherwise, the cell thicknesses and locations of local extrema change little, primarily because the electromagnetic damping, proportional to the radial flow velocity, increases as the flow intensifies.

The primary flow instabilities for small-Pr liquid bridges with magnetic stabilization are hydrodynamic in nature. The first instability for $\operatorname{Pr}<0.4$ is characterized by stationary disturbances [Bouizi et al. 2007]. Within this range, [Houchens and Walker 2005] further suggested three subregimes with different axial symmetries of the perturbations, which were confirmed in [Bouizi et al. 2007], both in full-zone geometries. In [Levenstam and Amberg 1995; Leypoldt et al. 2000] the secondary instability was found to be three-dimensional and oscillatory in the half-zone.

For high-Pr liquid bridges, convective heat transfer is dominant over conduction and the instability mechanism is hydrothermal. The base flow first transitions to oscillatory perturbations (see [Leypoldt et al. 2000], for example). Due to strong thermal convection at high Pr, isotherms are dramatically distorted such that a large temperature gradient exists at the free surface near the top and bottom boundaries. In reality, the melt-solid interfaces may deform significantly from the assumed rigid plane due to this strong thermal convection. To accurately resolve high-Pr liquid bridges, more realistic boundary conditions are needed, hence this work is limited to low-Pr cases.

For a fixed $\operatorname{Pr}>0$ (with $\operatorname{Re}_{\mathrm{FZ}}$ fixed as in Figure 5), increasing Ha continuously confines the flow into a narrower region near the free surface and reduces the effectiveness of convective heat transfer into the interior. Thus $T_{\max }$ increases, which enhances the thermocapillary driving effect. Therefore the flow

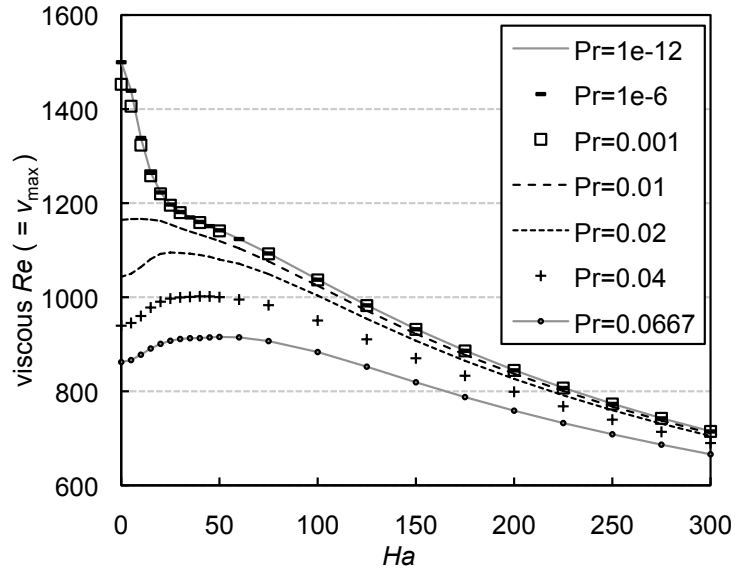

(a) $\operatorname{Re}_{\text {viscous }}$

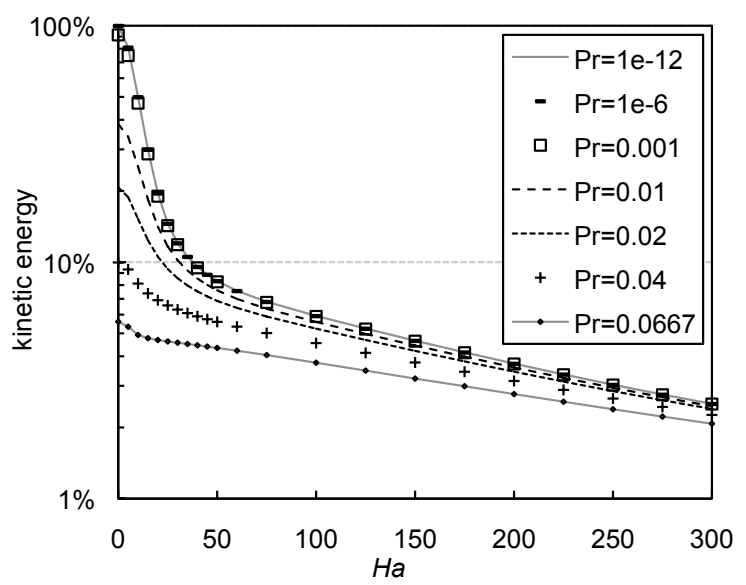

(b) Nondimensional kinetic energy (semilog)

Figure 5. Effects of Prandtl number and magnetic damping on viscous Reynolds number and nondimensional kinetic energy $\left(\operatorname{Re}_{\mathrm{FZ}}=21,914.8\right.$ fixed in all cases). 
velocity increases at the free surface, but this is limited to a narrow cell due to magnetic damping. $\operatorname{Re}_{\text {viscous }}$ also increases proportionally to the maximum nondimensional velocity $v_{\max }$ which always occurs near $(r, z)=(1, b)$. This explains the initial increase of $\mathrm{Re}_{\text {viscous }}$ with $\mathrm{Ha}$ for $\operatorname{Pr} \geq 0.02$ in Figure 5a. Eventually magnetic damping is sufficiently large that $v_{\max }$ is reduced, even though $T_{\max }$ continues to increase and approach 1.0633 (the value in the limit of no convective heat transfer). Also observe that $\mathrm{Re}_{\text {viscous }}$ decreases monotonically with increasing $\mathrm{Ha}$ for $\operatorname{Pr} \leq 0.001$. For $\operatorname{Pr} \leq 0.001$ the temperature field is effectively decoupled from the flow field and $T_{\max }$ remains constant as Ha increases. Therefore increasing Ha does not enhance the thermocapillary effect. $\operatorname{Pr}=0.01$ marks the division between these two trends.

The nondimensional kinetic energy, computed as the integral of velocity squared over the domain, drops quickly as Ha increases (see Figure $5 b$ ), which demonstrates the magnetic damping effect. For a fixed $\operatorname{Re}_{\mathrm{FZ}}\left(\propto q^{*} R^{*} / k^{*}\right)$, the fact that kinetic energy also drops as $\operatorname{Pr}\left(=\mu^{*} c_{p}^{*} / k^{*}\right)$ increases can be explained as follows. Assume that the dynamic viscosity $\mu^{*}$, specific heat $c_{p}^{*}$, and radius $R^{*}$ remain unchanged. Increasing Pr then corresponds to decreasing the thermal conductivity $k^{*}$ and consequently a reduction in maximum heat flux $q^{*}$ (to maintain a constant $\operatorname{Re}_{\mathrm{FZ}}$ ). Therefore with less thermocapillary driving force, kinetic energy within the melt is reduced as $\operatorname{Pr}$ increases. Also note that the $\operatorname{Pr}=0.001$, $\operatorname{Pr}=10^{-6}$ and $\operatorname{Pr}=10^{-12}$ curves are virtually indistinguishable, which indicates that $\operatorname{Pr}=0.001$ is a good approximation for the limit of $\operatorname{Pr} \rightarrow 0$, at least over the range $0 \leq \mathrm{Ha} \leq 300$.

\section{Normal-mode linear stability analysis}

4A. Disturbances. Normal-mode linear stability analysis, which compares well with nonlinear simulations in the half-zone [Levenstam et al. 2001], was used to study the stability of the base flow in the full-zone liquid bridge. The base flow was subjected to infinitesimal three-dimensional normal-mode disturbances of the form

$$
\begin{array}{ll}
\xi(r, \theta, z, t)=\xi_{0}(r, z)+\varepsilon \operatorname{Real}\left\{\exp (\lambda t+i m \theta) \xi_{1}(r, z)\right\} & \text { for } \xi=v_{r}, v_{z}, P, T, j_{\theta}, \\
\zeta(r, \theta, z, t)=\varepsilon \operatorname{Real}\left\{\exp (\lambda t+i m \theta) i \zeta_{1}(r, z)\right\} & \text { for } \zeta=v_{\theta}, \phi, j_{r}, j_{z},
\end{array}
$$

where $\varepsilon$ is an infinitesimal magnitude and $m$ denotes the azimuthal wave number of the disturbance. For uniqueness, disturbance waves must complete themselves as they travel through $\theta=0 \rightarrow 2 \pi$, therefore $m$ is integer valued. Axisymmetric $m=0$ cases were not investigated as they have been shown to be very stable in similar systems [Kasperski et al. 2000]. The extra factor of $i$ in (4-2) accounts for the phase shift in the variables which are zero in the base state, yielding a purely real linear stability problem.

Disturbances adhere to one of two axial symmetries. When perturbation variables have the same axial symmetry as their corresponding base flow variables, the mode is denoted as "symmetric". When perturbation variables have the opposite axial symmetry as their corresponding base flow variables, the mode is denoted as "antisymmetric". Any combination of these disturbance types will result in a critical $\operatorname{Re}_{\mathrm{FZ}}$ that larger than the smaller $\mathrm{Re}_{\mathrm{FZ}, \mathrm{cr}}$ of these two.

Figure 6 shows an example of streamlines in the base flow along with the disturbed flow. In the axisymmetric base flow state, a weightless fluid particle released at the starting point circulates on the black closed path (the stream function contour) within a fixed $\theta$ plane. This specific example first transitions to stationary antisymmetric disturbances with $m=2$. At the critical $\mathrm{Re}_{\mathrm{FZ}}$, a particle released at the same starting point changes its "orbiting radius" as it circulates, while oscillating within a $\theta=\pi / m$ wedge. 

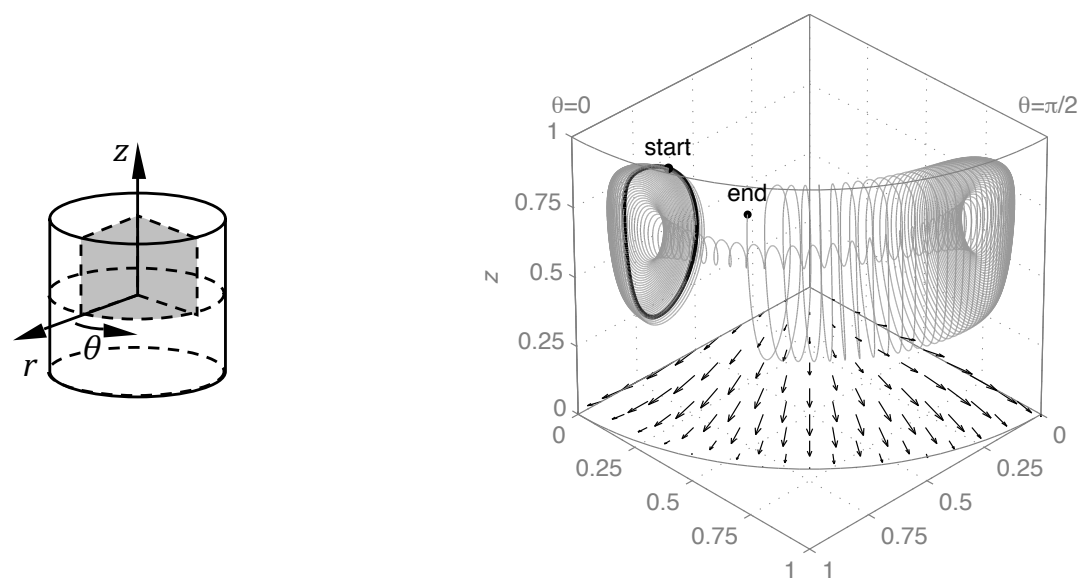

Figure 6. Streamlines in the base flow (black closed path) and disturbed flow (gray spiral path), and disturbed flow (vectors) at the midplane $\left(\mathrm{Pr}=0.02, \mathrm{Ha}=0\right.$ at $\operatorname{Re}_{\mathrm{FZ}, \mathrm{cr}}=$ 1546.58, stationary antisymmetric disturbances with $m=2$, base flow grid $r \times z=35 \times 45$, temperature grid $r \times z=30 \times 30$, linear stability grid $r \times z=28 \times 28, \alpha=300$, perturbation versus base flow maximum magnitude ratio $5 \%$ ).

Note that the path is cut off intentionally at the "end" point to better show its spiral structure. Flow in other wedges and in the lower half of the liquid bridge can be inferred from the symmetry and mode number of the disturbance.

Arrows in the $z=0$ plane of Figure 6 show the disturbed flow motion at the liquid bridge midplane. Flow circulates from the liquid bridge interior back to the free surface due to the viscous effect. The flow at $z=0$ has no azimuthal component due to the antisymmetric disturbance mode. Perturbed flow crosses the midplane into the upper or lower half of the liquid bridge. In contrast, the half-zone assumes a no-slip, no-penetration midplane.

4B. Critical thermocapillary Reynolds number. The critical $\mathrm{Re}_{\mathrm{FZ}, \mathrm{cr}}$ measures the critical point at which the base flow transitions to the first instability. Beyond $\mathrm{Re}_{\mathrm{FZ}, \mathrm{cr}}$, one or more infinitesimal disturbances grow in time, breaking either the axisymmetry or the axial symmetry or both, and potentially evolving the motion to a periodic flow. The goal is to find the first transition to instability among all possible disturbance modes.

By substituting the disturbance variables of (4-1) and (4-2) into the governing equations and boundary conditions and linearizing (neglecting $\varepsilon^{2}$ terms) and discretizing, a generalized eigenvalue problem is obtained:

$$
\underline{\underline{A M}} \underline{x}=\lambda \underline{\underline{B M}} \underline{x}
$$

The entire generalized system was solved using either the routine RGG in EISPACK or the routine DGGEV in LAPACK [Anderson et al. 1999], with refinement of the critical eigenvectors performed via the inverse iteration method [Saad 1992]. The real part of the leading eigenvalue (or pair) $\lambda_{R}$ determines the stability of this system. If the imaginary part of the leading eigenvalue pair $\lambda_{I} \neq 0$, the base flow transitions to a periodic disturbance. Otherwise $\lambda_{I}=0$ and the transition is stationary. For each disturbance 
mode ( $\mathrm{Pr}, \mathrm{Ha}, m$, antisymmetric or symmetric mode), a neutrally stable mode is found if the leading eigenvalue (or pair) has $\lambda_{R}=0$. A slight increase in $\mathrm{Re}_{\mathrm{FZ}}$ causes this mode to become unstable and grow in time.

Finding the $\mathrm{Re}_{\mathrm{FZ}}$ associated with a neutrally stable mode is an iterative process. Starting from a stable $\operatorname{Re}_{\mathrm{FZ}, s}$ (with $\lambda_{R}<0$ ) and an unstable $\operatorname{Re}_{\mathrm{FZ}, u}$ (with $\lambda_{R}>0$ ), a better approximation $\operatorname{Re}_{\mathrm{FZ}, 3}$ is predicted using the regula falsi method. The new $\lambda_{R}$ corresponding to $\operatorname{Re}_{\mathrm{FZ}, 3}$ is computed using the shifted inverse iteration method. The $\mathrm{Re}_{\mathrm{FZ}, 3}$ then replaces either $\mathrm{Re}_{\mathrm{FZ}, s}$ or $\mathrm{Re}_{\mathrm{FZ}, u}$. In this way the bounds on the neutrally stable $\mathrm{Re}_{\mathrm{FZ}}$ are narrowed through iterations until convergence. The linear stability analysis code is written in Fortran 90.

Variables in the linear stability analysis are the velocity perturbations $v_{r 1}$ and $v_{z 1}$, temperature perturbation $T_{1}$, and electric potential perturbation $\phi_{1}$. Reduction from the 9 primitive stability variables to these four is accomplished using incompressible continuity and $\theta$ momentum to solve for $v_{\theta 1}$ and $P_{1}$, respectively. The electric current density perturbations $\left(j_{r 1}, j_{\theta 1}\right.$, and $\left.j_{z 1}\right)$ are solved using Ohm's law and then substituted into conservation of charge. The resulting set of partial differential equations can be found in [Houchens and Walker 2001] for the half-zone. Similarly to the base flow analysis, these variables are represented as Chebyshev polynomials. Only even or odd terms in both $r$ and $z$ are nonzero due to the symmetries. In addition, by modeling antisymmetric and symmetric disturbance modes separately, the full-zone liquid bridge domain can be halved at the midplane. The price is that two codes have to be developed. An $N_{R} \times N_{Z}$ Gauss-Lobatto collocation grid is adopted for $r \in(0,1)$ and $z \in(0, b)$, which is equivalent to a grid resolution of $r \times z=\left(2 \times N_{R}\right) \times\left(4 \times N_{Z}\right)$ in the fulldomain liquid bridge simulation if no symmetries are observed. The finest stability analysis grid used was $N_{R} \times N_{Z}=50 \times 70$ for high-Ha cases.

Disturbances with a wide range of azimuthal wave numbers $m$, for both the antisymmetric and symmetric modes, are investigated. Axisymmetric disturbances $(m=0)$ are not studied in this work because they are unlikely to be the critical disturbance mode. For example [Bouizi et al. 2007] reported that $\operatorname{Re}_{\mathrm{FZ}, \mathrm{cr}}$ for the $m=0$ mode ranges from seven times to thousands of times larger than $\mathrm{Re}_{\mathrm{FZ}, \mathrm{cr}}$ for the critical $m=2$ mode for $\operatorname{Pr} \in(0.001,0.04)$.

For a fixed Pr, a neutrally stable $\mathrm{Re}_{\mathrm{FZ}}$ versus Ha branch can be obtained for each disturbance mode. The critical $\mathrm{Re}_{\mathrm{FZ}, \mathrm{cr}}$ is the lowest among all neutrally stable $\mathrm{Re}_{\mathrm{FZ}}$ 's. For example, the neutral stability branches for $\operatorname{Pr}=0.02$ (Figure 7a) show how the critical disturbance mode changes from $m=2$ to 3 and then 4 for $\mathrm{Ha} \in(0,50)$. The $m=2$ symmetric branch (dashed line) deviates from the critical antisymmetric modes as Ha increases. Although other stability branches above the critical branch are not valid for predicting a second and third bifurcation, they provide insight into flow stability when subject to these perturbation modes.

Table 1 lists $\operatorname{Re}_{\mathrm{FZ}}$ values on the $\mathrm{Pr}=0.02$ neutral stability branches. For both antisymmetric and symmetric disturbance modes, $m$ is tracked from 1 to 8 for $\operatorname{Pr}=0.02$, and to at least the critical $m+4$ for $\operatorname{Pr}=0.001$. $\operatorname{Re}_{\mathrm{FZ}}$ values not shown in Table 1 are higher than these listed $\mathrm{Re}_{\mathrm{FZ}}$ 's for the same Ha. The $m=5$ antisymmetric branch never becomes critical up to $\mathrm{Ha}=300$ for $\mathrm{Pr}=0.02$.

The $\mathrm{Re}_{\mathrm{FZ}, \text { cr }}$ versus $\mathrm{Ha}$ curves for $\mathrm{Pr}=0.02$ and $\mathrm{Pr}=0.001$ (see Figure $7 \mathrm{~b}$ ) summarize the first instabilities over a wide Ha range. The $\mathrm{Re}_{\mathrm{FZ}, \mathrm{cr}}$ increases quickly with increasing Ha, which demonstrates the magnetic stabilization effect. Stronger magnetic fields damp the flow, so that more driving energy must be fed in before the flow trips to an instability. The critical curves determine the minimum magnetic 


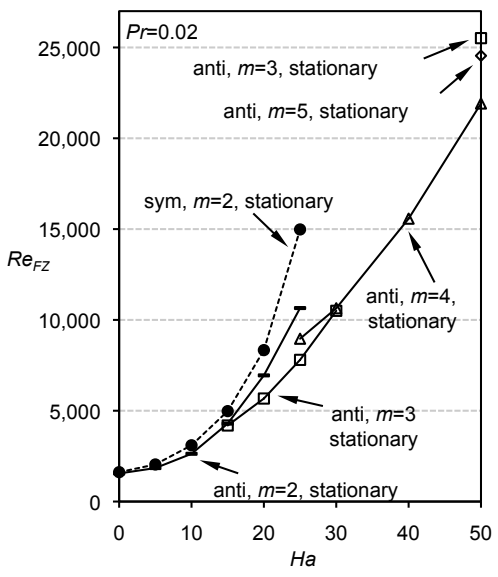

(a) Neutral stability branches

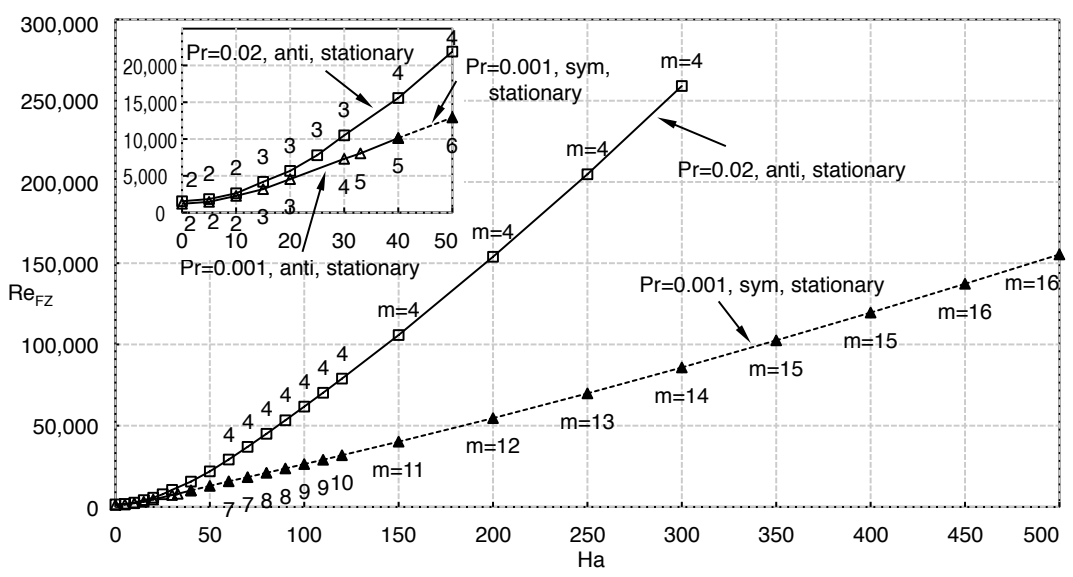

(b) First instability $\mathrm{Re}_{\mathrm{FZ}, \text { cr }}$ versus Ha curves

Figure 7. Example of neutral stability branches for $\operatorname{Pr}=0.02$ and the first instability critical curves for $\operatorname{Pr}=0.02$ and for $\operatorname{Pr}=0.001$.

\begin{tabular}{|c|c|c|c|c|c|}
\hline \multirow[b]{2}{*}{$\mathrm{Ha}$} & \multicolumn{4}{|c|}{ Antisymmetric } & \multirow{2}{*}{$\begin{array}{c}\text { Symmetric } \\
m=2\end{array}$} \\
\hline & $m=2$ & $m=3$ & $m=4$ & $m=5$ & \\
\hline 0 & $1,546.58$ & & & & $1,618.42$ \\
\hline 5 & $\overline{1,842.97}$ & & & & $2,041.81$ \\
\hline 10 & $2,629.52$ & & & & $3,098.39$ \\
\hline 15 & $4,291.10$ & $4,187.74$ & & & $4,978.35$ \\
\hline 20 & $6,946.57$ & $\overline{5,671.88}$ & & & $8,337.20$ \\
\hline 25 & $10,653.30$ & 7,800.11 & $8,964.73$ & & $14,980.91$ \\
\hline 30 & & $1 \overline{0,501.11}$ & $10,642.13$ & & \\
\hline 40 & & & $\underline{15,575.20}$ & & \\
\hline 50 & & $25,513.47$ & $21,914.83$ & $24,545.29$ & \\
\hline 60 & & & $\underline{29,168.06}$ & $30,544.57$ & \\
\hline 70 & & & $\underline{36,961.68}$ & $37,672.75$ & \\
\hline 80 & & & $44,999.40$ & $45,250.00$ & \\
\hline 90 & & & $\overline{53,316.88}$ & $53,583.48$ & \\
\hline 100 & & & $61,747.38$ & $62,263.88$ & \\
\hline 110 & & & $70,289.58$ & $71,229.01$ & \\
\hline 120 & & & $\underline{79,037.36}$ & $80,562.15$ & \\
\hline 150 & & & $1 \overline{05,776.82}$ & $109,765.83$ & \\
\hline 200 & & $162,032.06$ & $\overline{153,945.51}$ & & \\
\hline 250 & & & $204,740.95$ & $225,107.28$ & \\
\hline 300 & & $263,317.80$ & $259,068.47$ & $293,492.96$ & \\
\hline
\end{tabular}

Table 1. Neutrally stable $\operatorname{Re}_{\mathrm{FZ}}$ 's for $\operatorname{Pr}=0.02$ branches ( $\mathrm{Re}_{\mathrm{FZ}, \mathrm{cr}}$ 's are underlined). 
field strength required to avoid instabilities in the optically heated float-zone crystal growth process. Note that $\operatorname{Pr}=0.001$ is chosen because its critical curve very likely marks the lower limit of critical curves for $\operatorname{Pr} \rightarrow 0$. According to [Houchens and Walker 2005], at $\mathrm{Ha}=0$ the $\mathrm{Re}_{\mathrm{FZ}, \mathrm{cr}}$ for $\operatorname{Pr}=10^{-10}$ is less than $1 \%$ smaller than $\operatorname{Re}_{\mathrm{FZ}, \mathrm{cr}}$ for $\operatorname{Pr}=0.001$. Details of the perturbation flow field and the energy analysis between the base state and perturbed field are presented and validated with other liquid bridge studies in [Huang and Houchens 2011]. Here the focus is instead on the numerical issues that arise in the spectral collocation technique and linear stability analyses. The following sections are widely applicable to a range of partial differential equations. First, identification of spurious eigenvalues is discussed. Then, regularization and grid dependence issues are covered. Finally, the use of pseudospectra to investigate the robustness of stability analyses are addressed.

4C. Identifying spurious eigenvalues. In the linear stability analysis, unstable systems are identified by positive leading eigenvalues. However, some spurious eigenvalues (usually with very large magnitudes) emerge in the generalized eigenvalue problem, (4-3), bearing no physical meaning regarding system stability. It is crucial to identify and separate them from the remaining legitimate eigenvalues to correctly predict the stability of the system.

Legitimate eigenvalues are independent of linear stability grid resolution. In Table 2, to test if the leading eigenvalue pair $350.47 \pm 7542.09 i$ on a $30 \times 40$ grid is legitimate or spurious, the linear stability code was run on two other grid sizes, $28 \times 28$ and $40 \times 50$. The fact that $350.47 \pm 7542.09 i$ is not present on these two grids indicates that it is a spurious eigenmode. All other leading eigenvalues agree well, independent of the grid.

As a direct proof, perturbation variable contours are plotted (Figure 8a) using eigenvectors corresponding to the spurious eigenvalue pair $350.47 \pm 7542.09 i$. The checkerboard pattern has no physical justification, but is rather an oscillation of a high-order mode(s) in each direction between Gauss-Lobatto collocation points. This is clearly shown in the Chebyshev polynomial coefficient plot (Figure $8 b$ ) for $v_{r 1}$, where

$$
v_{r 1}\left(r, \frac{z}{b}\right)=r^{m-1} \sum_{L=0}^{N_{R}+1} \sum_{M=0}^{N_{Z}} \mathscr{A}_{L M} T_{2 L}(r) T_{2 M}\left(\frac{z}{b}\right) .
$$

\begin{tabular}{|cllc|}
\hline Grid size & $r \times z=28 \times 28$ & \multicolumn{1}{c}{$r \times z=30 \times 40$} & $r \times z=40 \times 50$ \\
& & $\frac{350.47 \pm 7542.09 i}{}$ & \\
& $-34.61 \pm 9.25 i$ & $-34.61 \pm 9.26 i$ & $-34.61 \pm 9.25 i$ \\
Leading & $-94.58 \pm 110.75 i$ & $-94.58 \pm 110.75 i$ & $-94.58 \pm 110.75 i$ \\
eigenvalues & $-113.45 \pm 56.60 i$ & $-113.46 \pm 56.59 i$ & $-113.46 \pm 56.60 i$ \\
& $-129.35 \pm 175.31 i$ & $-129.35 \pm 175.31 i$ & $-129.35 \pm 175.31 i$ \\
& $-140.05 \pm 72.15 i$ & $-140.04 \pm 72.15 i$ & $-140.04 \pm 72.15 i$ \\
& $-197.10 \pm 320.75 i$ & $-197.10 \pm 320.75 i$ & $-197.10 \pm 320.75 i$ \\
& $\vdots$ & $\vdots$ & $\vdots$ \\
\hline
\end{tabular}

Table 2. Identification of a spurious eigenvalue (underlined) through grid refinement of the linear stability analysis $\left(\operatorname{Pr}=0.001, \mathrm{Ha}=0, \operatorname{Re}_{\mathrm{FZ}}=1000, m=1, \alpha=400\right.$, symmetric mode). 


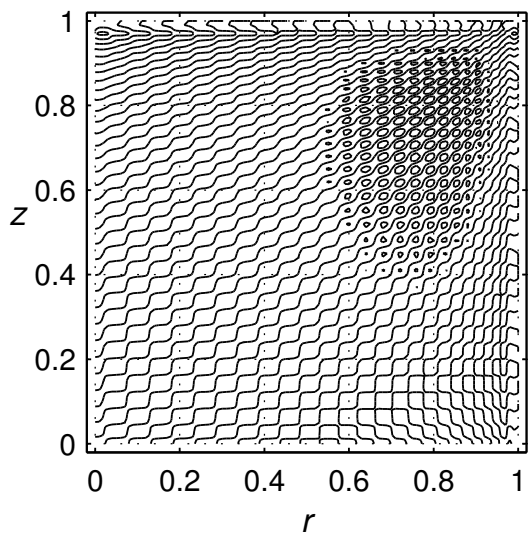

(a) $v_{r 1}$ contours

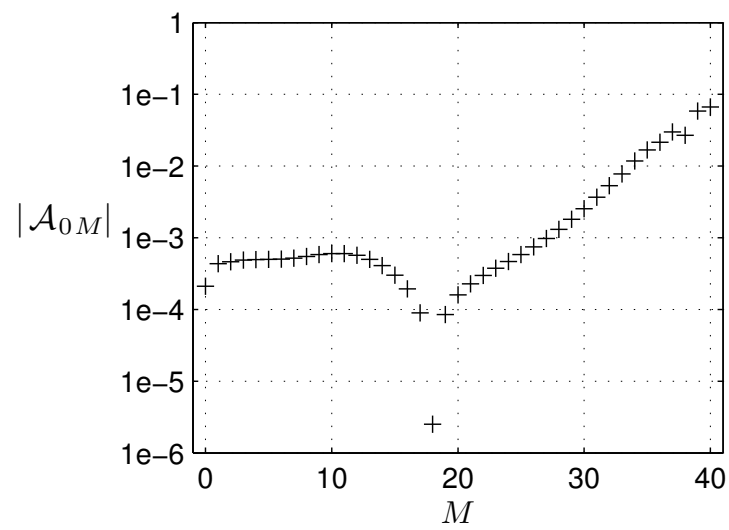

(b) Chebyshev polynomial coefficients for $v_{r 1}$

Figure 8. Perturbation contours and Chebyshev polynomial coefficients associated with the spurious eigenmode $350.47 \pm 7542.09 i$ in Table $2\left(\mathrm{Pr}=0.001, \mathrm{Ha}=0, \mathrm{Re}_{\mathrm{FZ}}=1000\right.$, $m=1, \alpha=400$, linear stability grid $r \times z=30 \times 40)$.

The coefficients of the highest-order Chebyshev terms have the largest magnitudes, which are responsible for the high-frequency, oscillating contours. Note that for a legitimate eigenmode, the Chebyshev polynomial coefficients decay exponentially when the representation is sufficient. Therefore, for this case the legitimate leading eigenvalue is a complex pair with negative real part $-34.61 \pm 9.26 i$ as shown in Table 2. The base flow is stable at $\operatorname{Re}_{\mathrm{FZ}}=1000$ for this branch $(\operatorname{Pr}=0.001, \mathrm{Ha}=0$, and $m=1$ symmetric disturbance mode).

\section{Numerical aspects}

5A. Regularization of the vorticity singularity. A regularization function $F(z)$ (from (3-7)) is introduced in the thermocapillary boundary condition to remove the singularity of the velocity gradient at the corner $(r, z)=(1, b)$. While $\partial v_{z 0} / \partial r=0$ at $(r, z)=(1, b)$ due to the boundary condition $v_{z 0}=0$ at $z=b$, the thermocapillary boundary condition, without regularization, gives a nonzero $\partial v_{z 0} / \partial r$ at $(r, z)=(1, b)$ due to the nonzero temperature gradient $\partial T_{0} / \partial z$ at the free surface. This singularity is removed by multiplying the thermocapillary boundary condition by a function that decays quickly to 0 as $z \rightarrow b$ but remains equal or close to 1 for the rest of $z$.

An optimum value of the regularization parameter $\alpha$ is achieved when increasing $\alpha$ further has no measurable impact on the flow and the singularity is effectively removed. Figure 9 shows a test over a wide range of $\alpha$. With a sufficiently large value the physics becomes independent of $\alpha$, as indicated by the "desired range". But too large an $\alpha$ may provide insufficient regularization. In Figure 9, the $v_{r 0}$ contours bear wiggles and circles, indicating $\alpha=50,000$ is too large for this case. With even less smoothing (higher $\alpha$ ), the vorticity singularity may cause the numerical solver to predict the wrong physics, suggested by the sudden drop of the critical $\operatorname{Re}_{\mathrm{FZ}}$ beyond $\alpha=100,000$. On the other hand, if the value of $\alpha$ is too small, the regularization effectively reduces the heat input at the free surface which reduces the driving force and explains the increase of the critical $\operatorname{Re}_{\mathrm{FZ}}$ ("too much regularization") for $\alpha$ in range 10-100 in Figure 9. 


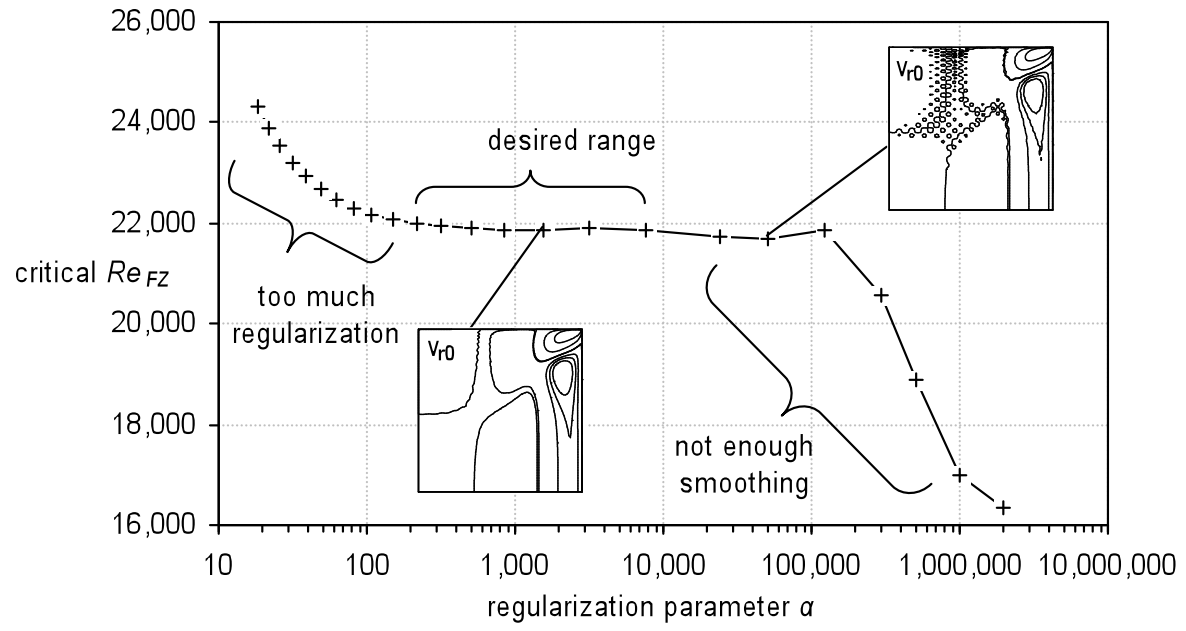

Figure 9. Searching for the desired range of regularization parameter $\alpha$ (semilog plot, $\operatorname{Pr}=0.02, \mathrm{Ha}=50$, flow grid $r \times z=40 \times 40$, temperature grid $r \times z=30 \times 30$, stability grid $r \times z=40 \times 40$ ).

A fast-decaying regularization function is especially important for high-Pr liquid bridge simulation. Due to strong heat convection, the temperature at the free surface varies slowly along $r=1$ until very close to $(r, z)=(1, b)$. This feature is best preserved by a regularization function that decays quickly near $(r, z)=(1, b)$. The choice of regularization function is arbitrary as long as the singularity is removed with minimum modification of the physics. For example, in [Bouizi et al. 2007] a power function regularization function $F(z)=\left(1-z^{2 n}\right)^{2}$ was adopted, where $n$ is a regularization parameter.

5B. Grid resolution and independence. A solution which does not vary with significant further grid refinement indicates that the grid resolution is sufficient and the results are reliable. Table 3 shows such a test based on the critical $\mathrm{Re}_{\mathrm{FZ}}$. Significant resolution increases are introduced for the base flow grids and the stability analysis grid, but the $\mathrm{Re}_{\mathrm{FZ} \text {,cr }}$ barely changes, demonstrating grid independence was achieved.

More subtle inferences can be made from this test. $\operatorname{Re}_{\mathrm{FZ}, \mathrm{cr}}$ increased slightly on finer grids in Table 3 . This is expected because a fixed regularization parameter $(\alpha=400)$ affects more grid points on a finer axial grid than on a coarser axial grid. The thermocapillary effect is weakened at more collocation

the critical $\operatorname{Re}_{\mathrm{FZ}}$ for

flow grid, temperature grid $(r \times z)$ $30 \times 75,25 \times 25$ $70 \times 100,40 \times 40$ stability grid $(r \times z)$ $34 \times 60 \quad 50 \times 70$

$61,775.37 \quad 61,840.74$

$61,789.51 \quad 61,841.79$

$0.02 \% \quad 0.002 \%$

$\%$ difference

Table 3. Grid dependence study based on $\mathrm{Re}_{\mathrm{FZ}, \mathrm{cr}}$ for $\operatorname{Pr}=0.02, \mathrm{Ha}=100, \alpha=400$. 
points on finer grids, therefore a higher $\mathrm{Re}_{\mathrm{FZ}, \mathrm{cr}}$ is expected. However if this regularization effect were eliminated, $\operatorname{Re}_{\mathrm{FZ}, \mathrm{cr}}$ would be lower on finer grids, because coarser grids tend to smear out the largest gradients, which contribute energy to the instability mechanism. On coarser grids, $\operatorname{Re}_{\mathrm{FZ}, \mathrm{cr}}$ increases to compensate for this smearing effect until the energy is sufficient to trip the instability. Thus, in practice, it is necessary to carry out both grid and regularization dependence studies simultaneously. The regularization parameter must be increased as the grid is refined.

5C. Model robustness investigated by pseudospectra. As discussed above, the flow stability in the liquid bridge is determined by its eigenvalues. For the generalized eigenvalue problem, (4-3), each eigenvalue (each black dot in Figure 10) is associated with one flow perturbation eigenmode. Purely real eigenvalues correspond to perturbations in which the base flow transitions to steady, three-dimensional (not axisymmetric) perturbed flow. Complex eigenvalue pairs correspond to perturbations in which the base flow transitions to three-dimensional time-dependent flow, with the imaginary components representing the frequency.

Compared to the simplified numerical full-zone model, real world experiments include many imperfections that are difficult to represent. For example, in experiments the heat flux will not be strictly parabolic or axisymmetric and the free surface will not be exactly cylindrical. It is therefore desirable to predict what impact these imperfections might have, to verify the robustness of the model as compared to the experiment it is intended to represent.

Furthermore, numerical errors in the model may also play a significant but unpredictable role. For example, round-off errors in the eigenvalue problem may accumulate during computation and affect the results. Simulation results are more meaningful provided they are valid even when the model is subject

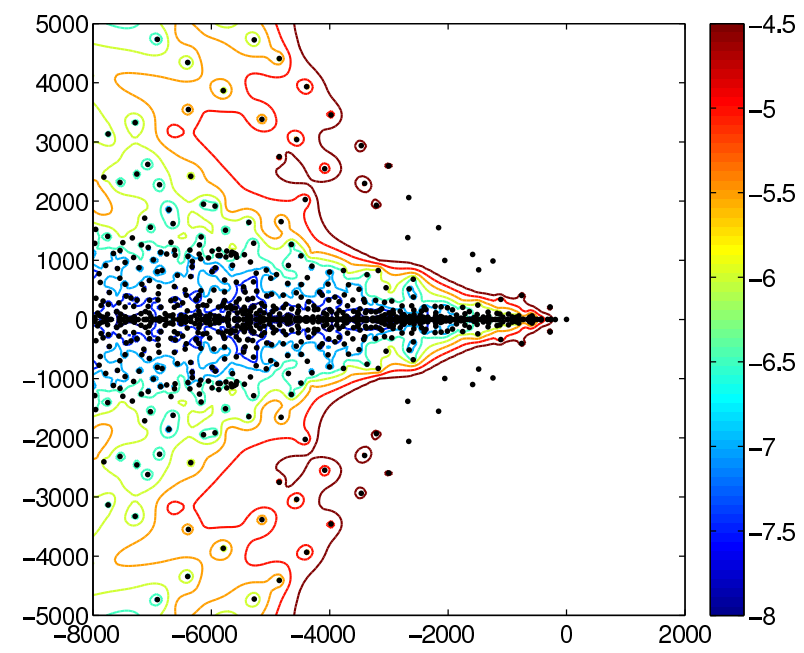

(a) Pseudospectra overview

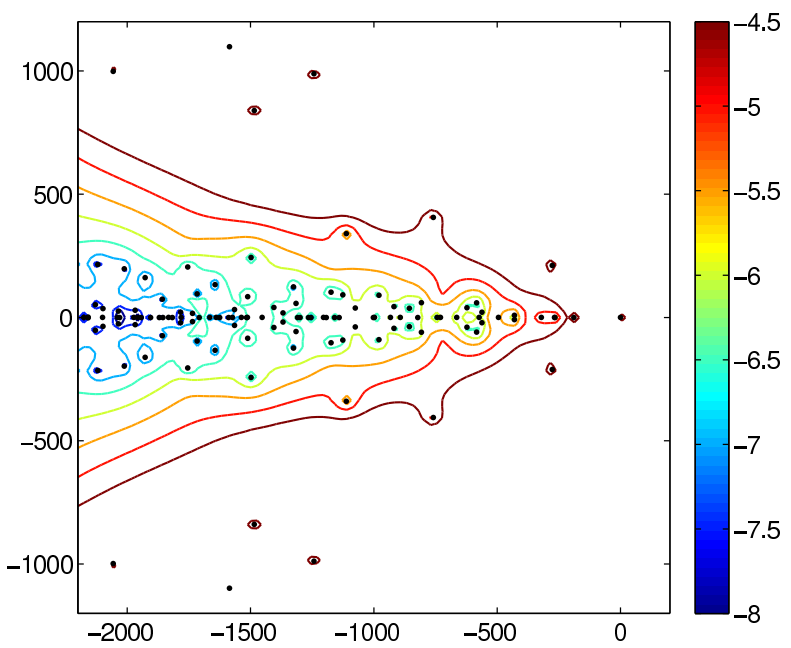

(b) Zoom in to the leading eigenvalues

Figure 10. Eigenvalues (black dots) and their pseudospectra $\sigma_{\epsilon}$ (contours) of the generalized eigenvalue problem (4-3) $\left(\mathrm{Pr}=0.02, \mathrm{Ha}=50, \mathrm{Re}_{\mathrm{FZ}}=21,879.5\right.$, flow grid $r \times z=70 \times 100$, temperature grid $r \times z=40 \times 40$, stability grid $r \times z=35 \times 50$, $\alpha=1000)$. 
to these minor changes, whether these are purely numerical or result from the inability of the model to represent small imperfections in the experiment.

Pseudospectral analysis provides a quantitative measure to test model robustness. Small numerical errors $\epsilon$ are purposefully introduced into either or both $\underline{\underline{A M}}$ and $\underline{\underline{B M}}$ matrices in the eigenvalue problem $\underline{\underline{A M}} \underline{\boldsymbol{x}}=\lambda \underline{\underline{B M}} \underline{x}$ (see (4-3)). Note that $\epsilon$ is different from the $\varepsilon$ used in the linear stability analysis. In fact, $\epsilon$ acts like a random perturbation on $\varepsilon$. The small errors introduced through $\epsilon$ can represent physical perturbations on the boundary conditions, geometry, and flow field of the model. They can also be purely numerical perturbations. In this problem such perturbations could be relatively minor (introducing slight curvature in the free surface) or more egregious (violations of conservation of charge).

After introducing these $\epsilon$ scale errors, the modified generalized eigenvalue problem is solved again to investigate any physically meaningful changes. For example, a possible scenario of significant interest would result if a previously more-stable branch became the critical mode in the modified system. This has tremendous relevance in this problem, as only one known experimental study in a low-Pr fluid in the half-zone configuration has indicated possible observation of the steady, three-dimensional transition before onset of periodicity [Takagia et al. 2001]. In other experiments, the instability has appeared to jump directly to the higher branch associated with the periodic transition, suggesting that the distinction between the preferred modes may be very subtle. Results will show, fortunately or unfortunately, that such sensitivity is not found in the full-zone model.

Introduced in [Reddy and Trefethen 1990], pseudospectra present a systematic method for carrying out such analyses. For an introduction and a simulation method (EigTool) for problems of small or moderate size, the reader is referred to [Embree and Trefethen 2011]. An extensive discussion on pseudospectra, including fluid mechanics applications, can be found in [Trefethen and Embree 2005].

For the large generalized eigenvalue problem in this work the matrix $\underline{\underline{\boldsymbol{A M}}}$ is perturbed with the random matrix $\underline{\underline{E}}$ in the way described in [van Dorsselaer 1997] using

$$
\begin{aligned}
\sigma_{\epsilon}(\underline{\underline{\boldsymbol{A M}}}, \underline{\underline{\boldsymbol{B M}}}) & =\left\{z \in \boldsymbol{C}:\left\|(z \underline{\underline{\boldsymbol{B M}}}-\underline{\underline{\boldsymbol{A M}}})^{-1}\right\|>1 / \epsilon\right\} \\
& =\{z \text { is an eigenvalue of }(\underline{\underline{\boldsymbol{A M}}}+\underline{\underline{\boldsymbol{E}}}, \underline{\underline{\boldsymbol{B M}}}) \text { for some } \underline{\underline{\boldsymbol{E}}} \text { with }\|\underline{\underline{\boldsymbol{E}}}\|<\epsilon\} .
\end{aligned}
$$

This analysis must be repeated until representative pseudospectra are obtained. Resulting pseudospectra $\sigma_{\epsilon}$ contours indicate the sensitivity of the original eigenvalues to the random noise introduced via $\epsilon$. The results of this analysis are given by the color contours in Figure 10. The color gradient indicates the magnitude of the $\epsilon$ perturbation. The smallest $\epsilon=10^{-8}$ perturbations do not affect the leading eigenvalues at a measurable level, hence the blue contours are not visible around these eigenvalues in the complex plane. The first blue envelope that can be observed at this order occurs for eigenvalues with real parts near -1500 . As the order of $\epsilon$ increases, more eigenvalues are influenced. For example, a perturbation of size $\epsilon=10^{-5}$ may move enclosed eigenvalues anywhere within the medium-red regions in Figure 10.

The leading real eigenvalue and complex eigenvalue pair are the most likely to become critical. The linear stability analysis predicts that the real eigenvalue will dominate. This is confirmed by the pseudospectra in Figure 10. Namely, at the largest pseudospectral perturbation of $\epsilon=10^{-4.5}$, the leading real eigenvalue maintains its leading position and explores only a very small region near its origin. In fact, no dark red contours of any eigenvalue extend into the positive half of the real plane. Since the pseudospectra 
contours of all the eigenvalues remain behind the leading eigenvalue, the branches associated with these modes will not become critical, even in the modified system. Therefore the critical eigenmode (stationary, antisymmetric disturbances with $m=4$ in this example) predicted by the original generalized eigenvalue problem remains valid under such perturbations. Therefore, in careful experiments it would be expected that the stationary mode would be observed first.

To quantitatively or qualitatively relate $\epsilon=10^{-4.5}$ numerical perturbations to specific physical changes in the fluid model is appealing, but intractable without imposing further structure on the perturbation. Random changes in matrix elements are often nonphysical, resulting in violations of the conservation of mass, energy, and momentum, and breaking the orthogonality of boundary conditions. Thus even a small $\epsilon$ may have significant impacts on the physical system. In fact, the entire domain in Figure 10 is contained in the contour $\sigma_{\epsilon}(\underline{\underline{\boldsymbol{M}}}, \underline{\underline{\boldsymbol{B M}}})$ for $\epsilon \geq 10^{-4.35}$. Nevertheless, the pseudospectra suggests the results of the linear stability analysis are quite robust, particularly for the leading eigenvalues which are of most interest.

\section{Conclusions}

A thermocapillary-driven full-zone liquid bridge with magnetic stabilization is modeled numerically. A wide range of three-dimensional flow disturbances are tracked using a normal-mode linear stability analysis. The first instability curve for Prandtl number $\operatorname{Pr}=0.02$ (for example, molten silicon) is obtained up to an intermediate Hartmann number of $\mathrm{Ha} \in(0,300)$. Within this range, the steady axisymmetric base flow first transitions to stationary three-dimensional disturbances with axial symmetries opposite to their base flow components (the antisymmetric disturbance mode). Moreover, first instabilities for $\operatorname{Pr}=0.001$, representing the $\operatorname{Pr} \rightarrow 0$ limit, are presented up to $\mathrm{Ha}=500$. For $\operatorname{Pr}=0.001$, the base flow also first transitions to stationary three-dimensional disturbances. Axial critical disturbance symmetries are antisymmetric below $\mathrm{Ha}=40$ and symmetric for $\mathrm{Ha} \in(40,500)$. The critical azimuthal wave number $m$ increases with $\mathrm{Ha}$ for both $\operatorname{Pr}=0.02$ and $\operatorname{Pr}=0.001$.

Magnetic stabilization effects are observed and quantitatively measured for a steady external magnetic field in the axial direction. The induced Lorentz force acts proportionally against radial flow motion, thus multiple cell-like circulation patterns form within the liquid bridge. The most significant flow is confined to an increasingly narrow region near the free surface as Ha increases. At the interior the flow is damped until it is almost stagnant, which provides steady crystal growth conditions at the interface. This damping effect is also confirmed by quantitative studies of the viscous Reynolds number and kinetic energy versus Ha. Because the flow disturbances are greatly suppressed by magnetic stabilization, a more intense thermocapillary driving force is needed to trip instabilities within the liquid bridge. For example, the critical thermocapillary Reynolds number $\mathrm{Re}_{\mathrm{FZ}, \mathrm{cr}}$ at $\mathrm{Ha}=300$ is two orders of magnitude larger than when no magnetic field $(\mathrm{Ha}=0)$ is applied.

Small-Prandtl number liquid bridges, dominated by heat conduction, are studied over the range $\operatorname{Pr} \in$ $\left(10^{-12}, 0.0667\right)$. For $\operatorname{Pr} \leq 0.001$, the temperature field is effectively decoupled from the flow field, mimicking a pure conduction state such that the temperature distribution is almost unchanged over the range $\mathrm{Ha}=0 \rightarrow 300$. Thermal convection becomes important as Pr increases. It helps unify the temperature distribution throughout the domain as suggested by the isotherms. At higher Pr, the temperature gradient on the free surface concentrates near the liquid-solid boundaries $(r, z)=(1, \pm b)$, which intensifies the 
thermocapillary effect in these regions. Interestingly, the flow "circulation cell" thicknesses and locations are almost unaffected by increases in Pr over the range studied, mainly because the electromagnetic force, proportional to the radial flow velocity, also increases as convection intensifies.

Numerical techniques and analyses are also highlighted extensively in this work. For example, the vorticity singularity in the spectral scheme is removed by applying an exponential regularization function. The desired range of the regularization parameter $\alpha$ is determined by balancing the removal of the singularity and the invariance of the physics. The generalized eigenvalue problem $\underline{\underline{A M}} \underline{x}=\lambda \underline{\underline{B M}} \underline{x}$ in the linear stability analysis is investigated from a numerical perspective. Spurious eigenvalue modes with no physical meaning are identified by a combination of grid dependence studies and plotting of the eigenmodes. The pseudospectra indicate that results predicted by this full-zone model are valid even if the model is subject to minor changes, be they numerical or physical.

Acknowledgements. The authors would like to thank Professor Mark Embree at Rice University for providing the pseudospectra plots and insights on the eigenvalue problem and pseudospectra analysis. All tests were run on the Shared University Grid at Rice (SUG@R) cluster. This work was partially supported by the United States Air Force Office of Scientific Research.

\section{References}

[Anderson et al. 1999] E. Anderson, Z. Bai, C. Bischof, S. Blackford, J. Demmel, J. Dongarra, J. Du Croz, A. Greenbaum, S. Hammarling, A. McKenney, and D. Sorensen, "Linear Algebra PACKage", 1999, available at http://www.netlib.org/lapack/ lug/index.html.

[Bouizi et al. 2007] O. Bouizi, C. Delcarte, and G. Kasperski, "Stability study of the floating zone with respect to the Prandtl number value", Phys. Fluids 19:11 (2007), 114102.

[Chen et al. 1997] G. Chen, A. Lizée, and B. Roux, "Bifurcation analysis of the thermocapillary convection in cylindrical liquid bridges", J. Cryst. Growth 180:3-4 (1997), 638-647.

[Cröll et al. 1994] A. Cröll, P. Dold, and K. W. Benz, "Segregation in Si floating-zone crystals grown under microgravity and in a magnetic field", J. Cryst. Growth 137:1-2 (1994), 95-101.

[Davis 1993] S. H. Davis, "Effects of flow on morphological stability", pp. 859-897 in Handbook of crystal growth, vol. 1B, edited by D. T. J. Hurle, Elsevier, New York, 1993.

[van Dorsselaer 1997] J. L. M. van Dorsselaer, "Pseudospectra for matrix pencils and stability of equilibria", BIT 37:4 (1997), 833-845.

[Embree and Trefethen 2011] M. Embree and L. N. Trefethen, "Pseudospectra gateway", 2011, available at http://www.comlab. ox.ac.uk/pseudospectra.

[Eyer et al. 1979] A. Eyer, R. Nitsche, and H. Zimmermann, "A double-ellipsoid mirror furnace for zone crystallization experiments in spacelab", J. Cryst. Growth 47:2 (1979), 219-229.

[Eyer et al. 1985] A. Eyer, H. Leiste, and R. Nitsche, "Floating zone growth of silicon under microgravity in a sounding rocket", J. Cryst. Growth 71:1 (1985), 173-182.

[Houchens and Walker 2001] B. C. Houchens and J. S. Walker, "Magnetic damping of the thermocapillary instability during floating-zone crystal growth in space", in Conference and exhibit on international space station utilization (Cape Canaveral, FL, 2001), AIAA, Reston, VA, 2001. Paper \#2001-5053.

[Houchens and Walker 2005] B. C. Houchens and J. S. Walker, "Modeling the floating zone: instabilities in the half zone and full zone", J. Thermophys. Heat Transf. 19:2 (2005), 186-198.

[Huang and Houchens 2011] Y. Huang and B. C. Houchens, "Magnetic stabilization, transition and energy analysis in the Marangoni driven full-zone at low Prandtl numbers", Eur. Phys. J. Spec. Top. 192:1 (2011), 47-62.

[Intel 2008] Intel, "Intel math kernel library", 2008, available at http://software.intel.com/en-us/intel-mkl. 
[Kasperski et al. 2000] G. Kasperski, A. Batoul, and G. Labrosse, "Up to the unsteadiness of axisymmetric thermocapillary flows in a laterally heated liquid bridge”, Phys. Fluids 12:1 (2000), 103-119.

[Lan and Yeh 2004] C. W. Lan and B. C. Yeh, "Three-dimensional simulation of heat flow, segregation, and zone shape in floating-zone silicon growth under axial and transversal magnetic fields", J. Cryst. Growth 262:1-4 (2004), 59-71.

[Lan and Yeh 2005] C. W. Lan and B. C. Yeh, "Effects of rotation on heat flow, segregation, and zone shape in a small-scale floating-zone silicon growth under axial and transversal magnetic fields", Fluid Dyn. Mater. Process. 1:1 (2005), 33-44.

[Lappa 2004] M. Lappa, "Combined effect of volume and gravity on the three-dimensional flow instability in noncylindrical floating zones heated by an equatorial ring”, Phys. Fluids 16:2 (2004), 331-443.

[Lappa 2005a] M. Lappa, "Analysis of flow instabilities in convex and concave floating zones heated by an equatorial ring under microgravity conditions", Comput. Fluids 34:6 (2005), 743-770.

[Lappa 2005b] M. Lappa, "Review: possible strategies for the control and stabilization of Marangoni flow in laterally heated floating zones", Fluid Dyn. Mater. Process. 1:2 (2005), 171-188.

[Levenstam and Amberg 1995] M. Levenstam and G. Amberg, "Hydrodynamical instabilities of thermocapillary flow in a half-zone", J. Fluid Mech. 297 (1995), 357-372.

[Levenstam et al. 2001] M. Levenstam, G. Amberg, and C. Winkler, "Instabilities of thermocapillary convection in a half-zone at intermediate Prandtl numbers", Phys. Fluids 13:4 (2001), 807-816.

[Leypoldt et al. 2000] J. Leypoldt, H. C. Kuhlmann, and H. J. Rath, "Three-dimensional numerical simulation of thermocapillary flows in cylindrical liquid bridges", J. Fluid Mech. 414 (2000), 285-314.

[Martinez and Eyer 1986] I. Martinez and A. Eyer, "Liquid bridge analysis of silicon crystal growth experiments under microgravity", J. Cryst. Growth 75:3 (1986), 535-544.

[Morthland and Walker 1996] T. E. Morthland and J. S. Walker, "Thermocapillary convection during floating-zone silicon growth with a uniform or non-uniform magnetic field", J. Cryst. Growth 158:4 (1996), 471-479.

[Nakamura et al. 1998] S. Nakamura, T. Hibiya, K. Kakimoto, N. Imaishi, S. Nishizawa, A. Hirata, K. Mukai, S. Yoda, and T. S. Morita, "Temperature fluctuations of the Marangoni flow in a liquid bridge of molten silicon under microgravity on board the TR-IA-4 rocket”, J. Cryst. Growth 186:1-2 (1998), 85-94.

[Prange et al. 1999] M. Prange, M. Wanschura, H. C. Kuhlmann, and H. J. Rath, "Linear stability of thermocapillary convection in cylindrical liquid bridges under axial magnetic fields", J. Fluid Mech. 394:1 (1999), 281-302.

[Reddy and Trefethen 1990] S. C. Reddy and L. N. Trefethen, "Lax-stability of fully discrete spectral methods via stability regions and pseudo-eigenvalues", Comput. Methods Appl. Mech. Eng. 80:1-3 (1990), 147-164.

[Saad 1992] Y. Saad, Numerical methods for large eigenvalue problems, Manchester University Press, Manchester, 1992.

[Takagia et al. 2001] K. Takagia, M. Otaka, H. Natsui, T. Arai, S. Yoda, Z. Yuan, K. Mukai, S. Yasuhiro, and N. Imaishi, "Experimental study on transition to oscillatory thermocapillary flow in a low Prandtl number liquid bridge", J. Cryst. Growth 233:1-2 (2001), 399-407.

[Trefethen and Embree 2005] L. N. Trefethen and M. Embree, Spectra and pseudospectra: the behavior of nonnormal matrices and operators, Princeton University Press, Princeton, NJ, 2005.

Received 30 Jun 2010. Revised 1 Mar 2011. Accepted 10 Apr 2011.

YUE HUANG: yue.huang@rice.edu

Department of Mechanical Engineering and Materials Science, Rice University, 6100 Main Street, Houston, TX 77005-1827, United States

BRENT C. Houchens: houchens@rice.edu

Department of Mechanical Engineering and Materials Science, Rice University, 6100 Main Street, Houston, TX 77005-1827, United States 


\title{
JOURNAL OF MECHANICS OF MATERIALS AND STRUCTURES
}

\author{
jomms.org
}

Founded by Charles R. Steele and Marie-Louise Steele

EDITORS

Charles R. SteEle

DAVIDE BIGONI

Stanford University, USA

YASUHIDE SHINDO

University of Illinois at Urbana-Champaign, USA

Tohoku University, Japan

\section{EDITORIAL BOARD}

$\begin{aligned} \text { H. D. BUI } & \text { École Polytechnique, France } \\ \text { J. P. CARTER } & \text { University of Sydney, Australia } \\ \text { R. M. CHRISTENSEN } & \text { Stanford University, USA } \\ \text { G. M. L. GLADWELL } & \text { University of Waterloo, Canada } \\ \text { D. H. HODGES } & \text { Georgia Institute of Technology, USA } \\ \text { J. HUTCHINSON } & \text { Harvard University, USA } \\ \text { C. HWU } & \text { National Cheng Kung University, Taiwan } \\ \text { B. L. KARIHALOO } & \text { University of Wales, UK } \\ \text { Y. Y. KIM } & \text { Seoul National University, Republic of Korea } \\ \text { Z. MROZ } & \text { Academy of Science, Poland } \\ \text { D. PAMPLONA } & \text { Universidade Católica do Rio de Janeiro, Brazil } \\ \text { M. B. RUBIN } & \text { Technion, Haifa, Israel } \\ \text { A. N. SHUPIKOV } & \text { Ukrainian Academy of Sciences, Ukraine } \\ \text { T. TARNAI } & \text { University Budapest, Hungary } \\ \text { F. Y. M. WAN } & \text { University of California, Irvine, USA } \\ \text { P. WRIGGERS } & \text { Universität Hannover, Germany } \\ \text { W. YANG } & \text { Tsinghua University, China } \\ \text { F. ZIEGLER } & \text { Technische Universität Wien, Austria } \\ & \\ \text { PRODUCTION } & \text { contact@ msp.org } \\ \text { SILVIO LEVY } & \text { Scientific Editor }\end{aligned}$

Cover design: Alex Scorpan

Cover photo: Mando Gomez, www.mandolux.com

See http://jomms.org for submission guidelines.

JoMMS (ISSN 1559-3959) is published in 10 issues a year. The subscription price for 2011 is US \$520/year for the electronic version, and \$690/year (+\$60 shipping outside the US) for print and electronic. Subscriptions, requests for back issues, and changes of address should be sent to Mathematical Sciences Publishers, Department of Mathematics, University of California, Berkeley, CA 94720-3840.

JoMMS peer-review and production is managed by EditFLow ${ }^{\circledR}$ from Mathematical Sciences Publishers.

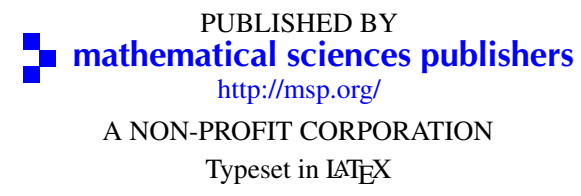

Copyright (C2011 by Mathematical Sciences Publishers 


\section{Journal of Mechanics of Materials and Structures}

\section{Volume 6, No. 7-8}

September-October 2011

\section{Special issue \\ Eleventh Pan-American Congress \\ of Applied Mechanics (PACAM XI)}

Preface

Adair R. Aguiar

949

Influence of specimen geometry on the Portevin-Le Châtelier effect due to dynamic strain aging

for the AA5083-H116 aluminum alloy

Rodrigo Nogueira de Codes and Ahmed Benallal

Dispersion relations for SH waves on a magnetoelectroelastic heterostructure with imperfect

interfaces

J. A. Otero, H. Calas, R. Rodríguez, J. Bravo, A. R. Aguiar and G. Monsivais

Numerical linear stability analysis of a thermocapillary-driven liquid bridge with magnetic stabilization

Yue Huang and Brent C. Houchens

Numerical investigation of director orientation and flow of nematic liquid crystals in a planar 1:4 expansion Pedro a. Cruz, Murilo F. Tomé, IAin W. Stewart and Sean McKee

Critical threshold and underlying dynamical phenomena in pedestrian-induced lateral vibrations of footbridges

Stefano LenCI and LAURA MARCHEgGiani

Free vibration of a simulation CANDU nuclear fuel bundle structure inside a tube

XUAN ZHANG and SHUdONG Yu

Nonlinear dynamics and sensitivity to imperfections in Augusti's model

D. Orlando, P. B. Gonçalves, G. Rega and S. LenCi

Active control of vortex-induced vibrations in offshore catenary risers: A nonlinear normal mode approach

CArlos E. N. MAZzilli and César T. SANCheS

Nonlinear electromechanical fields and localized polarization switching of piezoelectric macrofiber composites

Yasuhide Shindo, Fumio Narita, KoJi SATo and Tomo TAKeda

1089

Three-dimensional BEM analysis to assess delamination cracks between two transversely isotropic materials

Nicolás O. Larrosa, Jhonny E. Ortiz and Adrián P. Cisillino

Porcine dermis in uniaxial cyclic loading: Sample preparation, experimental results and modeling

A. E. Ehret, M. Hollenstein, E. MAzzA and M. Itskov

Analysis of nonstationary random processes using smooth decomposition

Rubens SAMpaio and Sergio Bellizzi

Perturbation stochastic finite element-based homogenization of polycrystalline materials

S. LePage, F. V. Stump, I. H. Kim and P. H. Geubelle

A collocation approach for spatial discretization of stochastic peridynamic modeling of fracture

Georgios I. Evangelatos and POL D. SPANOS 